\title{
2,3-Dihydro-1H-cyclopenta[b]quinoline Derivatives as Acetylcholinesterase Inhibitors—Synthesis, Radiolabeling and Biodistribution
}

\section{Pawel Szymański ${ }^{1, *}$, Alice Lázničková ${ }^{2}$, Milan Lázniček ${ }^{2}$, Marek Bajda ${ }^{3}$, Barbara Malawska $^{3}$, Magdalena Markowicz ${ }^{1}$ and Elżbieta Mikiciuk-Olasik ${ }^{1}$}

1 Department of Pharmaceutical Chemistry and Drug Analyses, Medical University, ul. Muszyńskiego 1, Lodz 90-151, Poland; E-Mails: magdalena.markowicz@umed.lodz.pl (M.M.); elzbieta.mikiciuk-olasik@umed.lodz.pl (E.M.-O.)

2 Faculty of Pharmacy in Hradec Kralove, Charles University in Prague, Heyrovskeho 1203, Hradec Kralove CZ-50005, Czech Republic; E-Mails: alice.laznickova@faf.cuni.cz (A.L.); milan.laznicek@faf.cuni.cz (M.L.)

3 Department of Physicochemical Drug Analysis, Faculty of Pharmacy, Jagiellonian University Medical College, Medyczna 9, Krakow 30-688, Poland; E-Mails: marek.bajda@uj.edu.pl (M.B.); barbara.malawska@uj.edu.pl (B.M.)

* Author to whom correspondence should be addressed; E-Mail: pawel.szymanski@umed.lodz.pl; Tel./Fax: +48-42-677-9250.

Received: 14 June 2012; in revised form: 7 July 2012 / Accepted: 6 August 2012 / Published: 13 August 2012

\begin{abstract}
In the present study we describe the synthesis and biological assessment of new tacrine analogs in the course of inhibition of acetylcholinesterase. The obtained molecules were synthesized in a condensation reaction between activated 6-BOC-hydrazinopyridine3-carboxylic acid and 8-aminoalkyl derivatives of 2,3-dihydro- $1 H$-cyclopenta[b]quinoline. Activities of the newly synthesized compounds were estimated by means of Ellman's method. Compound $\mathbf{6 h}\left(\mathrm{IC}_{50}=3.65 \mathrm{nM}\right)$ was found to be most active. All obtained novel compounds present comparable activity to that of tacrine towards acetylcholinesterase (AChE) and, simultaneously, lower activity towards butyrylcholinesterase (BChE). Apart from 6a, all synthesized compounds are characterized by a higher affinity for AChE and a lower affinity for $\mathrm{BChE}$ in comparison with tacrine. Among all obtained molecules, compound $6 \mathbf{h}$ presented the highest selectivity towards inhibition of acetylcholinesterase. Molecular modeling showed that all compounds demonstrated a similar binding mode with
\end{abstract}


AChE and interacted with catalytic and peripheral sites of AChE. Also, a biodistribution study of compound 6 a radiolabeled with ${ }^{99 \mathrm{~m}} \mathrm{Tc}$ was performed.

Keywords: biological activity; medicinal chemistry; isotopic labeling; drug design; radiopharmaceuticals

\section{Introduction}

Alzheimer's disease (AD), the most common form of dementia with an incidence that is highly age dependent, is a degenerative disease of the central nervous system characterized by a noticeable cognitive decline. The most characteristic symptoms of AD are memory loss, deficit in learning ability, and a reduced ability to perform the basic activities of daily living. With the ageing of the world population, the prevalence, cost, and potential societal effects of AD will increase with certainty.

Several theories explaining the mechanism of AD development have been proposed by scientists over the past decades. Loss of cholinergic function, known as cholinergic hypothesis, amyloid cascade (amyloid hypothesis), oxidative stress, decrease of steroid hormone concentration, and an inflammatory process are most often mentioned [1]. The widely accepted cholinergic hypothesis has been verified repeatedly.

The most characteristic abnormality associated with $\mathrm{AD}$ is a decrease in central cholinergic neurotransmission, a consequence of decreased activity of choline acetyltransferase (ChAT), an enzyme that synthesizes acetylcholine (ACh) [2,3]. Acetylcholine, an ester of choline and acetic acid, acts in both the peripheral nervous system and central nervous system. The second enzyme which influences the level of $\mathrm{ACh}$ is acetylcholinesterase (AChE). In its natural state, it is a monomer with a molecular weight of approximately $60 \mathrm{kDa}$; often, however, it forms aggregates which continue to produce catalytic activity. The enzyme monomer is an $\alpha / \beta$ protein that contains 537 amino acids. It consists of a 12-stranded mixed beta sheet surrounded by 14 alpha helices [4].

Recent scientific reports suggest that AChE also plays a non-cholinergic role in the development of $\mathrm{AD}[5,6]$ by working as a chaperone molecule, accelerating the $\mathrm{A} \beta$ peptide deposition, and the aggregation of $A \beta$ into insoluble fibrils [7,8].

One of the most popular therapeutic strategies in the treatment of $\mathrm{AD}$ is the control of cholinergic neurotransmission by slowing down the decline of neuronal degeneration or increasing cholinergic transmission [9]. Acetylcholinesterase inhibitors (AChEIs), which increase the synaptic amount of ACh by preventing its degradation, constitute the best-developed and widely approved class of AD drugs [10-13] and are used for mild-to-moderate stages of the disease. Results of clinical trials and imaging suggest that this group of drugs may lead also to a reduction in amyloid precursor protein (APP) formation [11]. Tacrine (1,2,3,4-tetrahydro-9-acridinamine-monohydrochloride) was the first drug approved by the United States Food and Drug Administration in 1993 for the palliative treatment of AD. It is a centrally active non-competitive reversible acetylcholinesterase inhibitor. However, its use is limited by a significant incidence of hepatotoxicity, cardiovascular system impairment, and mild cognitive benefits, while not altering the course of the disease [14]. 
Nevertheless, the search for new candidates-tacrine analogues is still of interest to scientists involved in AD research [15,16]. For example, 6-fluorotacrin-1-ol and 6-chlorotacrin-1-ol were found to be more potent than tacrine [17]. In the last decade, analogues containing two tacrine moieties linked by an alkylene chain were synthesized. It was reported that these dimeric molecules of tacrine were characterized by a stronger potency and a higher selectivity towards AChE $[18,19]$. It was proven that bis-(7)-tacrine had several modes of action, such as inhibition of AChE, $N$-Methyl-D-aspartate (NMDA) receptors, and nitric oxide synthase signaling. Furthermore, several homodimeric tacrine-based AChE inhibitors were synthesized. Their increased inhibitory potency was believed to be derived from the simultaneous binding of the units to the active and peripheral anionic sites of AChE [19]. One of these novel compounds, heptylene-linked bis-tacrine, was found to be 150-fold more active against rat $\mathrm{AChE}$ than tacrine and 250-fold more selective for acetylcholinesterase (AChE) than for butyrylcholinesterase (BChE) [20].

Second generation AChEIs (donepezil, rivastigmine and galantamine) demonstrate greater efficacy in AD treatment. Furthermore, when compared to tacrine, these drugs have fewer side effects and longer half-lives [6]. Therefore, synthesis of the analogues of the approved drugs: donepezil, rivastigmine, and galantamine is still of interest for many research groups [21,22].

Because of the complex pathophysiology of $\mathrm{AD}$, involving numerous pathways, development of a satisfactory therapy is problematic. The key therapeutic targets are diffuse loss of neurons, reduced levels of the neurotransmitter acetylcholine $(\mathrm{ACh})$, deposits of $\beta$-amyloid $(\mathrm{A} \beta)$ plaques, and neurofibrillary tangles [23]. For example, Tomassoli et al. reported that new 4-hydroxy-2-oxo-1,2-dihydroquinoline3-carboxamides, 4-hydroxy-2-oxo-1,2-dihydroquinoline-3-carbohydrazide, and hexahydropyrimido [5,4-c] quinoline-2,5-diones were characterized by moderate activity towards AChE/BuChE inhibition; none of the synthesized compounds, however, showed higher inhibitory activity than tacrine [24]. Another scientific team investigated the potential activity of substituted 2-aminopyridine-3-carbonitriles towards $\mathrm{AChE}$ and $\mathrm{BChE}$ inhibition. Biological studies showed that some of these molecules were good AChE inhibitors, in the nanomolar range, and quite selective with regards to the inhibition of $\mathrm{BChE}[25]$.

The purpose of this study was to synthesize new derivatives of 2,3-dihydro- $1 H$-cyclopenta $[b]$ quinoline and nicotinic acid and to determine their activity towards inhibition of AChE and BChE. Furthermore, the selectivity of synthesized compounds was determined. This is significant for further phases of our study related to complexation of radioactive isotopes because during the design of novel potential radiopharmaceuticals selectivity and capability of binding with specified biological targets are more important than activity. Molecular modeling studies for synthesized compounds were also performed in order to elucidate the interactions between the enzymes and synthesized compounds. Furthermore, one of the synthesized molecules (compound 6a) was labeled with ${ }^{99 \mathrm{~m}} \mathrm{Tc}$ and a biodistribution study of radioactivity was conducted following intravenous administration of ${ }^{99 \mathrm{~m}} \mathrm{Tc}-6 \mathrm{a}$ to rats in order to estimate its potential as diagnostic marker in AD. 


\section{Results and Discussion}

\subsection{Chemistry}

The first step of the synthesis was the preparation of 6-BOC-hydrazinopyridine-3-carboxylic acid (2). The substrate for this synthesis was 6-chloronicotinic acid. This compound reacted with hydrazine to give 6-hydrazinenicotinic acid (1). Subsequently, it was treated with di-tert-butyl dicarbonate and triethylamine in the presence of dimethylformamide (Scheme 1).

Scheme 1. Synthesis of 6-BOC-hydrazinopyridine-3-carboxylic acid. Reagents: (a) $85 \% \mathrm{NH}_{2} \mathrm{NH}_{2} ;$ (b) $(t-\mathrm{BuOCO})_{2} \mathrm{O}$, triethyl amine, DMF.<smiles>CC(=O)NNc1ccc(C(=O)O)cn1</smiles>

These syntheses were described earlier by Abrams and co-workers [26]. 8-chloro-2,3-dihydro- $1 \mathrm{H}$ cyclopenta[b]quinoline (3) was prepared according to a procedure mentioned in a previous paper [17]. This reaction involved cyclization of anthranilic acid with cyclopentanone in $\mathrm{POCl}_{3}$. Subsequently, the obtained compound (3) was coupled with the appropriate alkyldiamine (number of carbon atoms ranging from 2 to 9) and giving 8-amino-2,3-dihydro-1H-cyclopenta[b]quinolines (Scheme 2) [27-31].

Scheme 2. Synthesis of compounds (3), $\mathbf{4 a}-\mathbf{4 h}$. Reagents: (a) cyclopentanone, $\mathrm{POCl}_{3}$, reflux; (b) diamine, phenol, NaI, reflux.

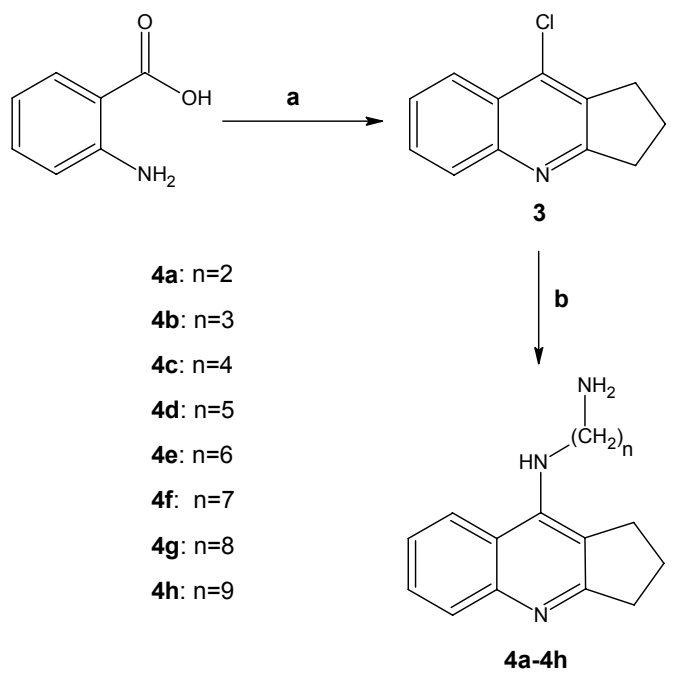


Novel compounds were obtained via the synthesis between 6-( $N$-tertbutoxycarbonylhydrazino)nicotinic acid (2), previously activated by 2-chloro-4,6-dimethoxy-1,3,5-triazine (CDMT), and $N$-methylmorpholine in solvent and reacted with compounds $\mathbf{4 a}-\mathbf{4 h}$. The best results (75\% yield) in this reaction were achieved with drop by drop addition of $N$-methylmorpholine to the solution in tetrahydrofuran at $-5{ }^{\circ} \mathrm{C}$. Monitoring the reaction by TLC showed that the reaction of activation of the carboxylic group was usually completed within 1-4 h; subsequently, a mixture of the appropriate reactant $4 \mathbf{a}-\mathbf{4 h}$ in the respective solvent was added at $-5{ }^{\circ} \mathrm{C}$. The last step of the synthesis involved conversion of the obtained compounds $\mathbf{5 a}-\mathbf{5 h}$ into hydrochlorides $\mathbf{6 a}-\mathbf{6 h}$ (Scheme 3 ) in the presence of hydrochloric acid with recrystallization from $\mathrm{HCl}$ in ether. In this step, the BOC group split off and the new compound precipitated.

Scheme 3. Synthesis of compounds $\mathbf{5 a}-\mathbf{5 h}$ and $\mathbf{6 a - 6 h}$. Reagents: (a) Reactant 2, 2-chloro4,6-dimethoxy-1,3,5-triazine (CDMT), $N$-methylmorpholine, THF; (b) $\mathrm{HCl} /$ ether.

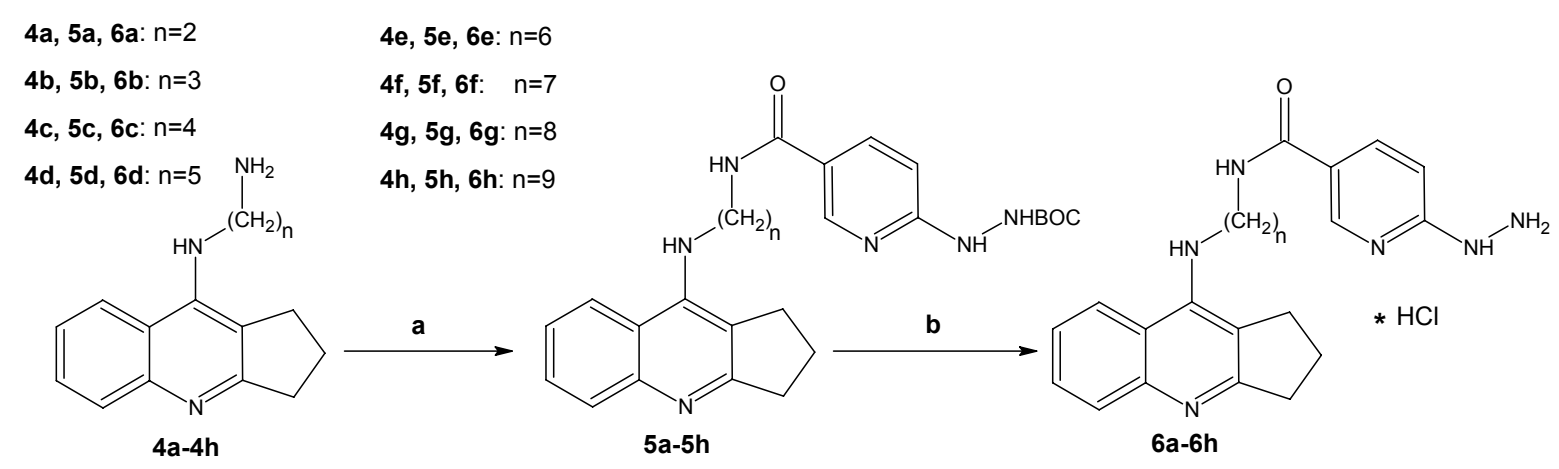

\subsection{Pharmacological Evaluation}

\subsubsection{Studies of AChE/BChE Inhibition}

The activity of the synthesized compounds towards inhibition of both enzymes (AChE and BChE) was estimated by Ellman's spectrophotometric method [32-34]. Table 1 presents $\mathrm{IC}_{50}$ values of the newly obtained compounds with respect to $\mathrm{AChE}$ and $\mathrm{BChE}$ inhibition. Among these molecules, the most active towards inhibition of $\mathrm{AChE}$ appears to be molecule $\mathbf{6 h}\left(\mathrm{IC}_{50}=3.65 \mathrm{nM}\right)$. Compound $\mathbf{6 g}$ $\left(\mathrm{IC}_{50}=5.17 \mathrm{nM}\right)$ exhibits a similar value of $\mathrm{IC}_{50}$ to tacrine. Obtained data shows that all synthesized molecules were characterized by lower BChE inhibitory activity in comparison with tacrine. Table 1 also lists values of relative inhibitory effects towards acetylcholinesterase (ratio $\mathrm{IC}_{50} \mathrm{BChE} / \mathrm{AChE}$ ) and butyrylcholinesterase (ratio $\mathrm{IC}_{50} \mathrm{AChE} / \mathrm{BChE}$ ). The most active molecule, compound $\mathbf{6 h}$, was more selective for AChE than tacrine. Derivative 6g, with similar activity with regard to tacrine, was characterized by a higher selectivity for $\mathrm{AChE}$ in comparison with tacrine. All acquired compounds possessed lower affinity for $\mathrm{BChE}$ than tacrine; among all synthesized compounds, compound 6a presented the highest activity for BChE. These findings are significant in view of the pathological processes involved in this type of neurodegenerative disease [35]. 
Table 1. $\mathrm{IC}_{50}$ values for activities towards acetylcholinesterase (AChE) and butyrylcholinesterase (BChE).

\begin{tabular}{ccccc}
\hline Compound & $\begin{array}{c}\left.\mathbf{A C h E ~ I C}_{\mathbf{5 0}}, \mathbf{( n M}\right) \\
\pm \mathbf{S E M}^{\mathbf{~}}\end{array}$ & $\begin{array}{c}\left.\mathbf{B C h E}_{\mathbf{I}} \mathbf{C}_{\mathbf{5 0}}, \mathbf{( n M}\right) \\
\pm \mathbf{S E M}^{\mathbf{b}}\end{array}$ & $\begin{array}{c}\text { Selectivity for } \\
\mathbf{A C h E}^{\mathbf{c}}\end{array}$ & $\begin{array}{c}\text { Selectivity for } \\
\mathbf{B C h E}^{\mathbf{d}}\end{array}$ \\
\hline $\mathbf{6 a}$ & $31.50 \pm 1.9$ & $164.00 \pm 1.6$ & 5.21 & 0.19 \\
$\mathbf{6 b}$ & $19.30 \pm 1.1$ & $197.00 \pm 3.4$ & 10.21 & 0.10 \\
$\mathbf{6 c}$ & $22.40 \pm 1.7$ & $2650.00 \pm 9.5$ & 118.30 & 0.01 \\
$\mathbf{6 d}$ & $7.84 \pm 2.2$ & $4240.00 \pm 2.2$ & 540.82 & 0.00 \\
$\mathbf{6 e}$ & $41.60 \pm 1.0$ & $16600.00 \pm 17.5$ & 399.04 & 0.00 \\
$\mathbf{6 f}$ & $17.60 \pm 0.8$ & $22700.00 \pm 20.6$ & 1289.77 & 0.00 \\
$\mathbf{6 g}$ & $5.17 \pm 1.4$ & $19600.00 \pm 16.4$ & 3791.10 & 0.00 \\
$\mathbf{6 h}$ & $3.65 \pm 0.5$ & $17100.00 \pm 21.3$ & 4684.93 & 0.00 \\
tacrine & $5.46 \pm 1.0$ & $2.44 \pm 0.6$ & 0.45 & 2.24 \\
\hline
\end{tabular}

${ }^{a}$ Inhibitor concentration (means \pm SEM of three experiments) for $50 \%$ inactivation of AChE;

b Inhibitor concentration (means \pm SEM of three experiments) for $50 \%$ inactivation of $\mathrm{BChE}$;

${ }^{\mathrm{c}}$ Selectivity for $\mathrm{AChE}$ is defined as $\mathrm{IC}_{50}(\mathrm{BChE}) / \mathrm{IC}_{50}(\mathrm{AChE}) ;{ }^{\mathrm{d}}$ Selectivity for $\mathrm{BChE}$ is defined as $\mathrm{IC}_{50}(\mathrm{AChE}) / \mathrm{IC}_{50}(\mathrm{BChE})$.

\subsubsection{Studies of Molecular Modeling}

A novel series of compounds was docked to acetyl- and butyrylcholinesterase to show the possible interactions between inhibitors and enzymes. All ligands demonstrated a similar binding mode with AChE. They were extended along the active gorge and interacted with catalytic and peripheral sites. The most active molecule (compound $\mathbf{6 h}$ ) and its binding mode are presented in Figure 1. The fragment of the tacrine analogue with a cyclopentane ring created a characteristic sandwich due to $\pi-\pi$ stacking with Trp84 and Phe330. The linker was located in the middle of the gorge, where it formed hydrophobic interactions with aromatic rings of Tyr121 and Tyr334. The nicotinamide moiety, in particular the amide bond, was located between two aromatic residues of the peripheral anionic site-Tyr70 and Trp279. Hydrazine interacted by H-bonding with the carbonyl group of the Asp276 backbone which was why the pyridine ring was shifted and was not able to create classical $\pi-\pi$ stacking; it was engaged in some hydrophobic interactions.

Figure 1. Binding mode of compound $\mathbf{6 h}$ with acetylcholinesterase.

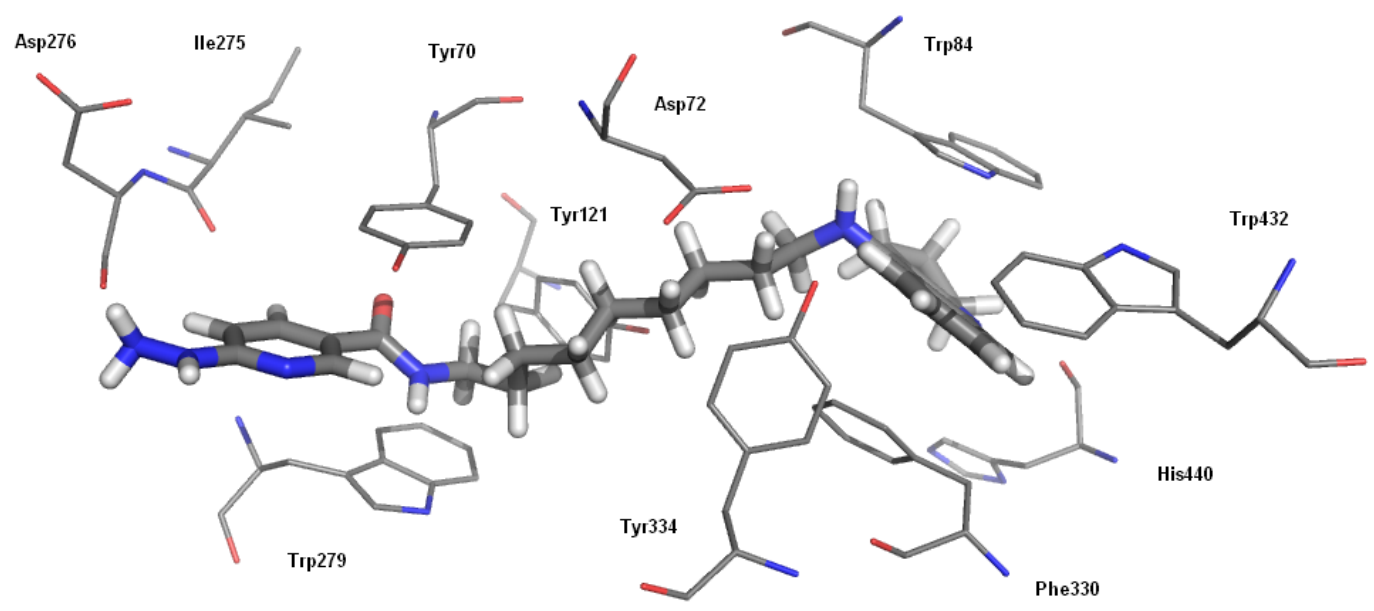


In the case of butyrylcholinesterase, the binding mode of the cyclopentaquinoline moiety was similar. The small differences concerned the location of the hydrazinenicotinic fragment in the reduced peripheral anionic site of $\mathrm{BChE}$. The binding mode of the most potent butyrylcholinesterase inhibitor 6a is shown in Figure 2. A fragment of the tacrine analogue created $\pi-\pi$ stacking with Trp82 and $\mathrm{CH}-\pi$ interactions with Trp430. The carbonyl group of the inhibitor amide bond formed $\mathrm{H}$-bonding with $\mathrm{OH}$ Thr120 and the hydrazine moiety with the $\mathrm{C}=\mathrm{O}$ of the Ile 69 backbone.

Figure 2. Binding mode of compound 6a with butyrylcholinesterase.

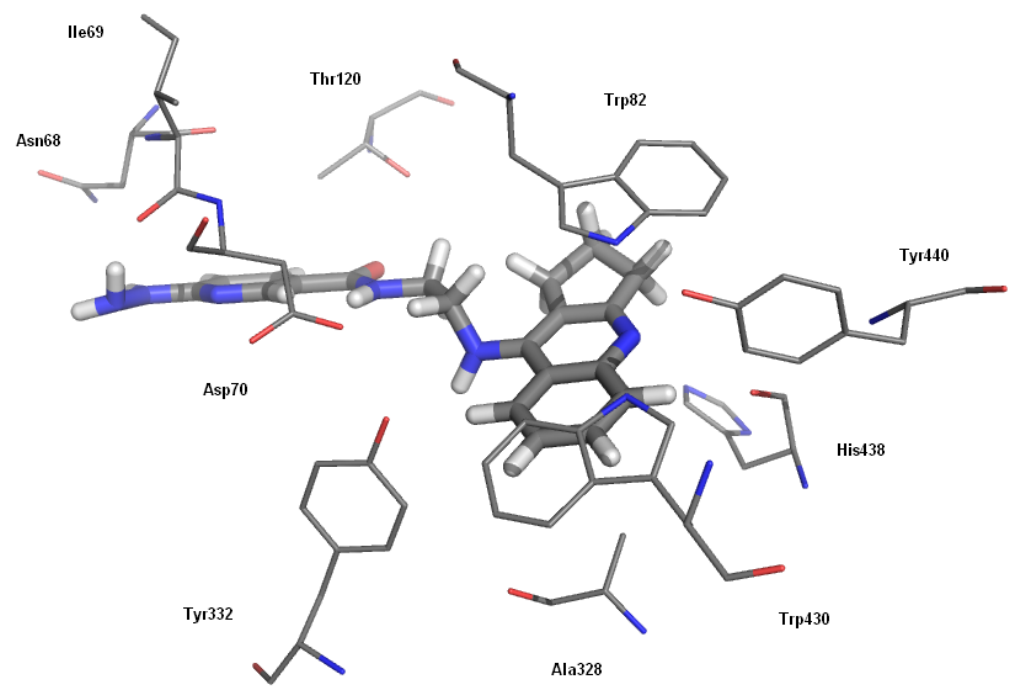

2.2.3. Radiolabeling with ${ }^{99 \mathrm{~m}} \mathrm{Tc}$ and Biodistribution Studies in Rats

Compound 6a was designed for radiolabeling. Also, spectrophotometric experiments were performed to determine its stability in water (Figure 3 ).

Figure 3. UV spectra of compound $\mathbf{6 a}$ in aqueous solution at different times of incubation ( 0 to $4 \mathrm{~h}$, measured every $15 \mathrm{~min}$ ).

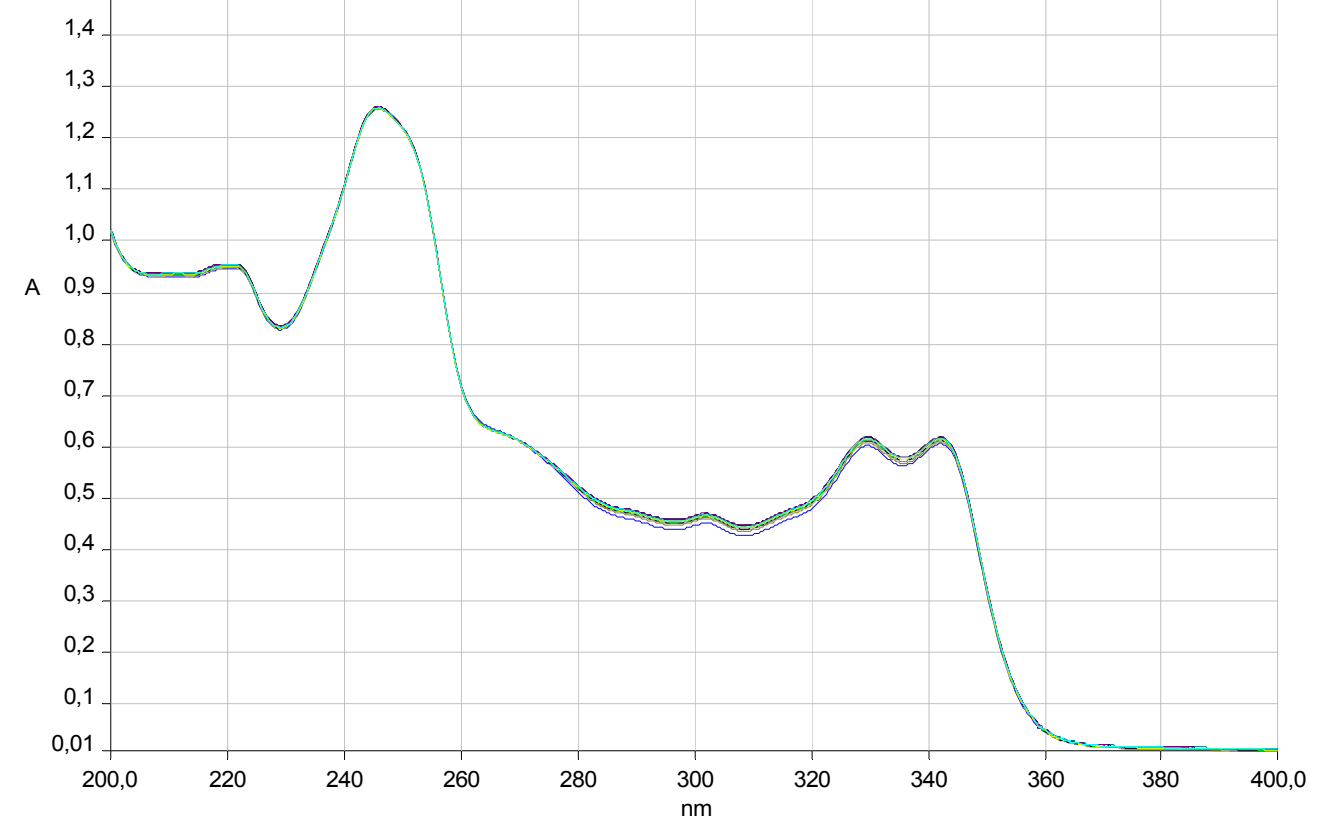


Taking into consideration all synthesized compounds, compound $\mathbf{6 a}$ is characterized by the highest selectivity towards BChE. Assessment of the level of this enzyme is very important as it is different in various stages of Alzheimer's disease. Quality control of ${ }^{99 m}$ Tc-labeled HYNIC-compounds with tricine as coligand with HPLC confirmed the purity of the product, which was found to be without unbound technetium (pertechnetate or hydrolyzed form, which would have appeared after a short elution time) [36]. Figure 4 presents radiochromatograms of complexes formed by technetium-99m with tricine and HYNIC.

Figure 4. Radiochromatograms of complexes formed by technetium-99m with tricine and hydrazine nicotinate (HYNIC). (a) Compound 6a; (b) Compound 6a after $24 \mathrm{~h}$.
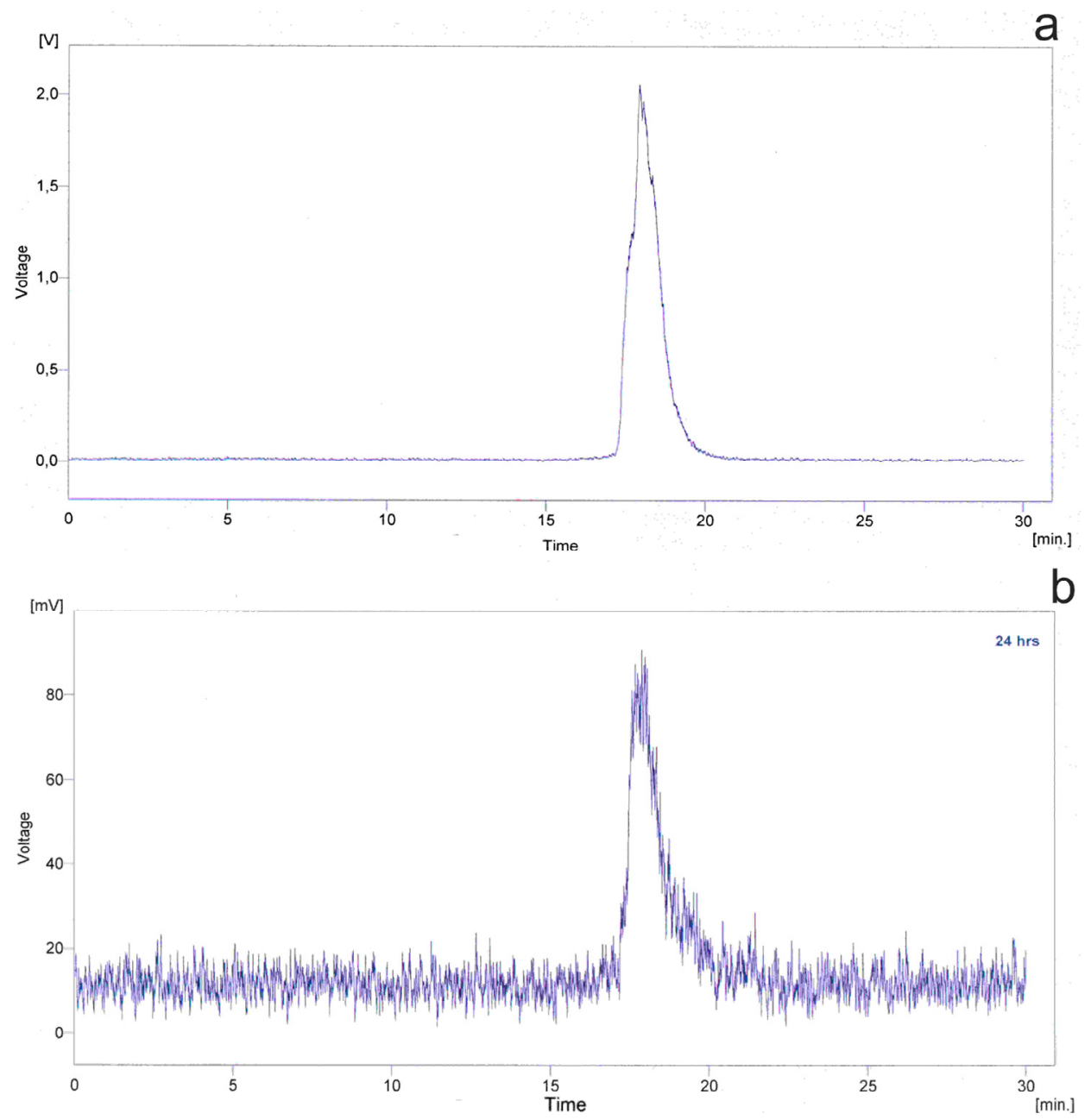

Table 2 presents the tissue distribution of radioactivity after intravenous administration of ${ }^{99 \mathrm{~m}} \mathrm{Tc}-6 \mathrm{a}$ to rats. Collectively, compound $\mathbf{6 a}$ exhibited relatively rapid blood radioactivity clearance; a large percentage of ${ }^{99 \mathrm{~m}}$ Tc-radioactivity was located in the liver, but also partly in the kidney, lung, and the gastrointestinal tract. Whereas the liver radioactivity uptake persisted for a long time for ${ }^{99 \mathrm{~m}} \mathrm{Tc}-6 \mathrm{a}$ (Table 2), radioactivity found in the kidney and the gastrointestinal tract is more likely connected with elimination of the parent compounds and/or their metabolites from the body. Radioactivity concentrations in the brain were very low; probably, as a result of the hydrophilicity of radiolabelled compounds (the effect of technetium and co-ligands attached to the 2,3-dihydro- $1 H$-cyclopenta 
[b]quinoline analogues), which are unable to cross the blood-brain barrier. This behavior is disadvantageous for the intended use of the agents for diagnosis of Alzheimer's disease.

Table 2. Distribution of radioactivity in selected organs and systems of rats after intravenous administration of ${ }^{99} \mathbf{m}$ Tc-6a.

\begin{tabular}{lcccc}
\hline \multicolumn{5}{c}{$\left.{ }^{\mathbf{9 9 m}} \mathbf{T c}-\mathbf{6 a} \mathbf{( \%} \mathbf{D o s e} / \mathbf{g}\right)$} \\
\hline Organs & $\mathbf{5} \mathbf{~ m i n}$ & $\mathbf{6 0} \mathbf{~ m i n}$ & $\mathbf{1 2 0} \mathbf{~ m i n}$ & $\mathbf{2 4} \mathbf{~ h}$ \\
\hline Blood & $0.850 \pm 0.108$ & $0.114 \pm 0.015$ & $0.091 \pm 0.017$ & $0.033 \pm 0.004$ \\
Plasma & $0.921 \pm 0.124$ & $0.183 \pm 0.024$ & $0.150 \pm 0.027$ & $0.052 \pm 0.003$ \\
Pancreas & $0.551 \pm 0.109$ & $0.117 \pm 0.019$ & $0.092 \pm 0.015$ & $0.067 \pm 0.006$ \\
Liver & $5.752 \pm 0.742$ & $5.160 \pm 0.586$ & $5.630 \pm 0.508$ & $5.095 \pm 0.478$ \\
Adrenals & $1.186 \pm 0.231$ & $0.900 \pm 0.102$ & $0.890 \pm 0.040$ & $0.793 \pm 0.137$ \\
Kidney & $3.838 \pm 0.264$ & $1.556 \pm 0.148$ & $1.332 \pm 0.209$ & $1.026 \pm 0.130$ \\
Lung & $3.497 \pm 0.239$ & $2.010 \pm 0.323$ & $1.824 \pm 0.438$ & $1.354 \pm 0.066$ \\
Heart & $0.779 \pm 0.078$ & $0.241 \pm 0.013$ & $0.204 \pm 0.025$ & $0.147 \pm 0.009$ \\
Spleen & $0.999 \pm 0.218$ & $1.840 \pm 0.618$ & $2.172 \pm 0.391$ & $2.631 \pm 0.199$ \\
Stomach & $0.206 \pm 0.044$ & $0.469 \pm 0.438$ & $0.311 \pm 0.153$ & $0.571 \pm 0.389$ \\
Intestine & $0.977 \pm 0.313$ & $4.242 \pm 0.113$ & $3.661 \pm 1.491$ & $0.196 \pm 0.055$ \\
Colon & $0.108 \pm 0.031$ & $0.044 \pm 0.003$ & $1.566 \pm 1.539$ & $1.809 \pm 0.976$ \\
Testes & $0.054 \pm 0.004$ & $0.033 \pm 0.001$ & $0.030 \pm 0.005$ & $0.022 \pm 0.003$ \\
Skin & $0.290 \pm 0.039$ & $0.196 \pm 0.017$ & $0.169 \pm 0.029$ & $0.100 \pm 0.010$ \\
Muscle & $0.157 \pm 0.004$ & $0.056 \pm 0.007$ & $0.048 \pm 0.006$ & $0.037 \pm 0.005$ \\
Thyroid & $0.551 \pm 0.040$ & $0.225 \pm 0.021$ & $0.189 \pm 0.009$ & $0.124 \pm 0.037$ \\
Brain & $0.049 \pm 0.003$ & $0.013 \pm 0.001$ & $0.014 \pm 0.003$ & $0.007 \pm 0.001$ \\
Fat & $0.305 \pm 0.060$ & $0.120 \pm 0.019$ & $0.105 \pm 0.017$ & $0.070 \pm 0.017$ \\
Femur & $0.330 \pm 0.036$ & $0.202 \pm 0.015$ & $0.218 \pm 0.031$ & $0.232 \pm 0.037$ \\
\hline
\end{tabular}

\section{Experimental Section}

\subsection{Chemistry}

During conducted syntheses dry organic solutions were used. This was achieved by employing anhydrous $\mathrm{Na}_{2} \mathrm{SO}_{4}$. Solvents were removed with a rotary evaporator. Melting points were determined by using an Electrothermal apparatus with open capillaries and were uncorrected. For monitoring of conducted reactions, TLC with 25 DC-Alufolien Kieselgel $60 \mathrm{~F}_{254}$ (Merck) was used; detection was carried out with a UV Lamp (254 nm). Column chromatography was executed using silica gel 60 (200-400 mesh, Merck). For determining IR spectra, Mattson Infinity Series FT-IR spectrophotometer was used. IR spectra were recorded in KBr. ${ }^{1} \mathrm{H}$ NMR spectra were recorded with Varian Mercury $300 \mathrm{MHz}$ spectrometer, with tetramethylsilane as an internal standard. Mass spectra were performed by the Centre of Molecular and Macromolecular Studies in Lodz (Polish Academy of Sciences). 


\subsubsection{6-Hydrazinopyridine-3-carboxylic Acid (1)}

6-Chloronicotinic acid $(8.0 \mathrm{~g}, 50.77 \mathrm{mmol})$ was dissolved in $80 \%$ hydrazine hydrate $(35 \mathrm{~mL}$, $930.0 \mathrm{mmol}$ ) and placed in a $100{ }^{\circ} \mathrm{C}$ oil bath for $4 \mathrm{~h}$. The homogeneous reaction mixture was cooled to room temperature and concentrated to dryness to give a white solid. The solid was dissolved in water and on acidification to $\mathrm{pH} 5.5$ with concentrated hydrochloric acid, a precipitate was formed. The precipitate was isolated by filtration; the solid was washed with $95 \%$ ethanol and ether, and dried in vacuum. Compound 1: yield 85\%; mp 292-293 ${ }^{\circ} \mathrm{C} ;{ }^{1} \mathrm{H}$ NMR (DMSO) $(\delta \mathrm{ppm}$.): $8.5(1 \mathrm{H}, \mathrm{s}, \mathrm{COOH})$, $8.3(1 \mathrm{H}, \mathrm{s}, \mathrm{CHN}), 7.8(1 \mathrm{H}, \mathrm{d}, J=2.4 \mathrm{~Hz} \mathrm{CCHC}), 6.7(1 \mathrm{H}, \mathrm{d}, J=8.8 \mathrm{~Hz}, \mathrm{CCHC}), 3.2(1 \mathrm{H}, \mathrm{s}, \mathrm{CNH}), 2.5$ $\left(2 \mathrm{H}, \mathrm{s}, \mathrm{NH}_{2}\right)$; IR $(\mathrm{KBr}) \vee\left(\mathrm{cm}^{-1}\right): 1333.0,3080.1,3308.2$; MS (FAB) $m / z(\mathrm{M}+1)$ 154.1; Elemental Analysis: Calc. for $\mathrm{C}_{6} \mathrm{H}_{7} \mathrm{~N}_{3} \mathrm{O}_{2}$ : C 47.06, H 4.61, N 27.44 Found: C 46.79 H 4.86 N 27.14.

\subsubsection{6-BOC-hydrazinopyridine-3-carboxylic Acid (2)}

To a solution of $1(1.4 \mathrm{~g}, 9.8 \mathrm{mmol})$ and triethylamine $(1.2 \mathrm{~mL}, 11.8 \mathrm{mmol})$ in DMF (10 mL) was added di-tert-butyl dicarbonate $(2.13 \mathrm{~g}, 9.8 \mathrm{mmol})$. The reaction mixture became homogeneous over $1 \mathrm{~h}$ and stirring was continued for $16 \mathrm{~h}$ at room temperature. The reaction mixture was concentrated to dryness under reduced pressure to give a brown solid. Recrystallization from ethyl acetate gave the desired product 2 as a white solid. Compound 2: yield 66\%; mp 282-285 ${ }^{\circ} \mathrm{C} ;{ }^{1} \mathrm{H}$ NMR (DMSO) ( $\delta$ ppm.): $12.5(1 \mathrm{H}, \mathrm{s}, \mathrm{COOH}), 8.9(1 \mathrm{H}, \mathrm{d}, J=2.4 \mathrm{~Hz} \mathrm{NHC}), 8.6(1 \mathrm{H}, \mathrm{s}, \mathrm{CHN}), 7.9(1 \mathrm{H}, \mathrm{d}, J=8.8 \mathrm{~Hz}$, CCHC), $6.5(1 \mathrm{H}, \mathrm{d}, J=8.8 \mathrm{~Hz} \mathrm{CCHC}), 3.3(1 \mathrm{H}, \mathrm{s}, \mathrm{CNH}), 1.9(9 \mathrm{H}, \mathrm{s}, \mathrm{BOC})$; IR $(\mathrm{KBr}) v\left(\mathrm{~cm}^{-1}\right)$ : 1608.8, 1706.4, 3253.7; MS (FAB) $m / z(\mathrm{M}+1)$ 254.2, 198.0; Elemental Analysis: Calc. for $\mathrm{C}_{11} \mathrm{H}_{15} \mathrm{~N}_{3} \mathrm{O}_{4}$ : C 52.17, H 5.97, N 16.59 Found: C 51.35, H 6.07, N 16.29.

\subsubsection{9-Chloro-2,3-dihydro-1H-cyclopenta[b]quinoline (3)}

To a mixture of anthranilic acid $(7.4 \mathrm{~g}, 53.9 \mathrm{mmol})$ and cyclopentanone $(1.64 \mathrm{~mL}, 53.9 \mathrm{mmol})$ was carefully added $30 \mathrm{~mL}$ of $\mathrm{POCl}_{3}$ in an ice bath. The mixture was heated under reflux for $2 \mathrm{~h}$, then cooled to room temperature, and concentrated under reduced pressure to give a slurry. The residue was diluted with ethyl acetate $(50 \mathrm{~mL})$, neutralized with aqueous $\mathrm{K}_{2} \mathrm{CO}_{3}(30 \mathrm{~mL})$, and washed with brine $(2 \times 20 \mathrm{~mL})$. The organic layer was dried over $\mathrm{MgSO}_{4}$ and concentrated to dryness under reduced pressure to give a brown solid. Recrystallization from acetone gave the desired product $\mathbf{1}$ as a yellow solid. Compound 1: yield 54\%; mp 85-87 ${ }^{\circ} \mathrm{C} ;{ }^{1} \mathrm{H}$ NMR $\left(\mathrm{CDCl}_{3}\right)(\delta \mathrm{ppm}):. 8.1(1 \mathrm{H}, \mathrm{d}, J=8.3 \mathrm{~Hz}$, $\operatorname{ArH}), 7.9(1 \mathrm{H}, \mathrm{d}, J=8.5 \mathrm{~Hz}, \mathrm{ArH}), 7.6(1 \mathrm{H}, \mathrm{t}, J=6.9 \mathrm{~Hz}, \mathrm{ArH}), 7.4(1 \mathrm{H}, \mathrm{t}, J=6.9 \mathrm{~Hz}, \mathrm{ArH}), 3.0(2 \mathrm{H}$, $\left.\mathrm{d}, J=5.9 \mathrm{~Hz}, \mathrm{CH}_{2}\right), 2.9\left(2 \mathrm{H}, \mathrm{d}, J=5.0 \mathrm{~Hz}, \mathrm{CH}_{2}\right), 1.8\left(2 \mathrm{H}, \mathrm{t}, J=6.9 \mathrm{CH}_{2}\right)$; IR $(\mathrm{KBr}) v\left(\mathrm{~cm}^{-1}\right): 766.2$, 1607.2, 2955.1, 2920.7, 3417.8 .

\subsection{4. $N^{\prime}$-(2,3-Dihydro-1H-cyclopenta[b]quinolin-9-yl)ethane-1,2-diamine (4a)}

A mixture of $3(0.71 \mathrm{~g}, 3.5 \mathrm{mmol}), 1,2$-diaminoethane $(0.47 \mathrm{~mL}, 7 \mathrm{mmol})$, phenol (1.5 g), and NaI $(0.07 \mathrm{~g})$ was carefully heated at $180{ }^{\circ} \mathrm{C}$ for $2 \mathrm{~h}$ and then cooled to room temperature. The mixture was diluted with ethyl acetate $(50 \mathrm{~mL})$ and made basic with $10 \% \mathrm{KOH}$ solution $(30 \mathrm{~mL})$. The organic layer was washed with water $(20 \mathrm{~mL})$ and brine $(2 \times 20 \mathrm{~mL})$, and then dried over $\mathrm{MgSO}_{4}$ and concentrated under reduced pressure. The resulting residue was purified with silica gel chromatography $\left(\mathrm{CH}_{2} \mathrm{Cl}_{2}\right.$ : 
$\left.\mathrm{CH}_{3} \mathrm{OH}: \mathrm{NH}_{3}=10: 4.6: 0.5\right)$ to afford $4 \mathbf{a}$ as an oil. Compound $4 \mathbf{a}$ : yield $64 \% ;{ }^{1} \mathrm{H}$ NMR $\left(\mathrm{CDCl}_{3}\right)$ ( $\delta$ ppm.): $7.8(1 \mathrm{H}, \mathrm{d}, J=8.3 \mathrm{~Hz}, \mathrm{ArH}), 7.7(1 \mathrm{H}, \mathrm{d}, J=8.3 \mathrm{~Hz}, \mathrm{ArH}), 7.5(1 \mathrm{H}, \mathrm{t}, J=6.9 \mathrm{~Hz}, \mathrm{ArH}), 7.3$ $(1 \mathrm{H}, \mathrm{t}, J=8.1 \mathrm{~Hz}, \mathrm{ArH}), 5.4(1 \mathrm{H}, \mathrm{s}, \mathrm{NH}), 3.6\left(2 \mathrm{H}, \mathrm{d}, J=5.4 \mathrm{~Hz}, \mathrm{NHCH}_{2}\right), 3.1(2 \mathrm{H}, \mathrm{t}, J=7.3 \mathrm{~Hz}$ $\left.\mathrm{CH}_{2} \mathrm{NH}_{2}\right), 2.8-3.0\left(4 \mathrm{H}, \mathrm{m}, \mathrm{CH}_{2}\right), 2.6\left(2 \mathrm{H}, \mathrm{p}, J=7.5,7.5 \mathrm{~Hz} \mathrm{CH}_{2}\right), 1.6\left(2 \mathrm{H}, \mathrm{s}, \mathrm{NH}_{2}\right), \mathrm{IR}(\mathrm{KBr}) v\left(\mathrm{~cm}^{-1}\right)$ : 1570.4, 2856.4, 2924.5, 2950.0, 3355.7; MS (FAB) $\mathrm{m} / z(\mathrm{M}+1)$ 228.1, 197.0, 185.0; MS-HR (FAB) Calc. for $\mathrm{C}_{14} \mathrm{H}_{17} \mathrm{~N}_{3}$ : 227.1422 Found: 227.14195.

\subsection{5. $N^{\prime}$-(2,3-Dihydro-1H-cyclopenta[b]quinolin-9-yl)propane-1,3-diamine (4b)}

A mixture of $3(0.71 \mathrm{~g}, 3.5 \mathrm{mmol})$, 1,3-diaminopropane $(0.58 \mathrm{~mL}, 7 \mathrm{mmol})$, phenol $(1.5 \mathrm{~g})$, and NaI $(0.07 \mathrm{~g})$ was combined as above to afford $\mathbf{4 b}$ as an oil. Compound $\mathbf{4 b}$ : yield $72 \%$; ${ }^{1} \mathrm{H}$ NMR $\left(\mathrm{CD}_{3} \mathrm{OD}\right)$ ( $\delta$ ppm.): $8.0(1 \mathrm{H}, \mathrm{d}, J=7.6 \mathrm{~Hz}, \mathrm{ArH}), 7.7(1 \mathrm{H}, \mathrm{d}, J=7.6 \mathrm{~Hz}, \mathrm{ArH}), 7.6(1 \mathrm{H}, \mathrm{t}, J=7.1 \mathrm{~Hz}, \mathrm{ArH}), 7.4$ $(1 \mathrm{H}, \mathrm{t}, J=6.8 \mathrm{~Hz}, \mathrm{ArH}), 3.7\left(2 \mathrm{H}, \mathrm{t}, J=7.1 \mathrm{~Hz}, \mathrm{NHCH}_{2}\right), 3.3\left(2 \mathrm{H}, \mathrm{t}, J=7.1 \mathrm{CH}_{2} \mathrm{NH}_{2}\right), 3.0(2 \mathrm{H}, \mathrm{t}$, $\left.J=7.1 \mathrm{~Hz}, \mathrm{CH}_{2}\right), 2.8\left(2 \mathrm{H}, \mathrm{t}, J=7.1 \mathrm{~Hz} \mathrm{CH}_{2}\right), 2.1\left(2 \mathrm{H}, \mathrm{p}, J=7.6,7.8 \mathrm{~Hz} \mathrm{CH}_{2}\right), 1.9(2 \mathrm{H}, \mathrm{p}, J=7.1,7.1 \mathrm{~Hz}$, $\mathrm{CH}_{2}$ ); IR (film) $v\left(\mathrm{~cm}^{-1}\right): 1568.8,2870.4,2951.9,3348.7$; MS (FAB) $\mathrm{m} / z(\mathrm{M}+1) 242.2,197.0,185.0$; MS-HR (FAB) Calc. for $\mathrm{C}_{15} \mathrm{H}_{19} \mathrm{~N}_{3}: 241.1579$ Found: 241.15789.

\subsection{6. $N^{\prime}$-(2,3-Dihydro-1H-cyclopenta[b]quinolin-9-yl)butane-1,4-diamine (4c)}

A mixture of 3 (0.71 g, $3.5 \mathrm{mmol})$, 1,6-diaminohexane (0.81 g, $7 \mathrm{mmol})$, phenol (1.5 g), and NaI $(0.07 \mathrm{~g})$ was combined as above to afford $4 \mathbf{c}$ as an oil. Compound $4 \mathbf{c}$ : yield $52 \% ;{ }^{1} \mathrm{H}$ NMR $\left(\mathrm{CD}_{3} \mathrm{OD}\right)$ ( $\delta$ ppm.): $8.0(1 \mathrm{H}, \mathrm{d}, J=7.6 \mathrm{~Hz}, \mathrm{ArH}), 7.7(1 \mathrm{H}, \mathrm{d}, J=7.3 \mathrm{~Hz}, \mathrm{ArH}), 7.5(1 \mathrm{H}, \mathrm{t}, J=6.8 \mathrm{~Hz}, \mathrm{ArH}), 7.4$ $(1 \mathrm{H}, \mathrm{t}, J=8.3 \mathrm{~Hz}, \mathrm{ArH}), 3.6\left(2 \mathrm{H}, \mathrm{t}, J=6.3 \mathrm{~Hz}, \mathrm{NHCH}_{2}\right), 3.2\left(2 \mathrm{H}, \mathrm{t}, J=7.1 \mathrm{CH}_{2} \mathrm{NH}_{2}\right), 2.9(2 \mathrm{H}, \mathrm{t}$, $\left.J=7.8 \mathrm{~Hz}, \mathrm{CH}_{2}\right), 2.7\left(2 \mathrm{H}, \mathrm{t}, J=7.1 \mathrm{~Hz} \mathrm{CH}_{2}\right), 2.1(2 \mathrm{H}, \mathrm{p}, J=7.6,7.6 \mathrm{~Hz} \mathrm{CH} 2), 1.6-1.8\left(4 \mathrm{H}, \mathrm{m}, \mathrm{CH}_{2}\right)$; IR (film) $v\left(\mathrm{~cm}^{-1}\right): 1566.8,2865.0,2934.1,3304.1$; MS (FAB) $m / z(\mathrm{M}+1)$ 256.2, 197.0, 185.0; MS-HR (FAB) Calc. for $\mathrm{C}_{16} \mathrm{H}_{21} \mathrm{~N}_{3}$ : 255.1735 Found: 255.17328.

\subsection{7. $N$-(2,3-Dihydro-1H-cyclopenta[b]quinolin-9-yl)penthane-1,5-diamine (4d)}

A mixture of $3(0.71 \mathrm{~g}, 3.5 \mathrm{mmol})$, 1,5-diaminopentane (0.82 $\mathrm{mL}, 7 \mathrm{mmol})$, phenol (1.5 g), and NaI $(0.07 \mathrm{~g})$ was combined as above to afford $\mathbf{4 d}$ as an oil. Compound $\mathbf{4 d}$ : yield $75 \% ;{ }^{1} \mathrm{H} \mathrm{NMR}\left(\mathrm{CDCl}_{3}\right)$ ( $\delta$ ppm.): $7.8(1 \mathrm{H}, \mathrm{d}, J=7.3 \mathrm{~Hz}, \mathrm{ArH}), 7.6(1 \mathrm{H}, \mathrm{d}, J=7.5 \mathrm{~Hz}, \mathrm{ArH}), 7.5(1 \mathrm{H}, \mathrm{t}, J=6.9 \mathrm{~Hz}, \mathrm{ArH}), 7.3$ $(1 \mathrm{H}, \mathrm{t}, J=6.9 \mathrm{~Hz}, \mathrm{ArH}), 4.6(1 \mathrm{H}, \mathrm{s}, \mathrm{NH}), 3.5\left(2 \mathrm{H}, \mathrm{m}, \mathrm{NHCH}_{2}\right), 3.1\left(2 \mathrm{H}, \mathrm{t}, J=6.9 \mathrm{~Hz}, \mathrm{CH}_{2}\right), 3.0(2 \mathrm{H}, \mathrm{t}$, $\left.J=7.7 \mathrm{~Hz}, \mathrm{CH}_{2} \mathrm{NH}_{2}\right), 2.6\left(2 \mathrm{H}, \mathrm{t}, J=6.7 \mathrm{~Hz}, \mathrm{CH}_{2}\right), 2.1\left(2 \mathrm{H}, \mathrm{p}, J=3.9,3.8 \mathrm{~Hz}, \mathrm{CH}_{2}\right), 1.9\left(2 \mathrm{H}, \mathrm{s}, \mathrm{NH}_{2}\right)$, $1.6\left(2 \mathrm{H}, \mathrm{p}, J=7.3,6.5 \mathrm{~Hz}, \mathrm{CH}_{2}\right), 1.3-1.5\left(4 \mathrm{H}, \mathrm{br}, \mathrm{CH}_{2}\right)$; IR (film) $v\left(\mathrm{~cm}^{-1}\right): 1567.9,2856.5,2931.8$, 3310.4; MS (FAB) $m / z(M+1)$ 270.3, 197.1, 185.0; MS-HR (FAB) Calc. for $\mathrm{C}_{17} \mathrm{H}_{23} \mathrm{~N}_{3}: 269.1892$ Found: $270.19712(\mathrm{M}+1)$.

\subsection{8. $N^{\prime}$-(2,3-Dihydro-1H-cyclopenta[b]quinolin-9-yl)hexane-1,6-diamine (4e)}

A mixture of 3 (0.71 g, $3.5 \mathrm{mmol})$, 1,6-diaminohexane (0.82 g, $7 \mathrm{mmol})$, phenol (1.5 g), and NaI $(0.07 \mathrm{~g})$ was combined as above to afford $4 \mathbf{e}$ as an oil. Compound $4 \mathbf{e}$ : yield $64 \% ;{ }^{1} \mathrm{H}$ NMR $\left(\mathrm{CD}_{3} \mathrm{OD}\right)$ $(\delta$ ppm.): $8.0(1 \mathrm{H}, \mathrm{d}, J=8.3 \mathrm{~Hz}, \operatorname{ArH}), 7.7(1 \mathrm{H}, \mathrm{d}, J=8.3 \mathrm{~Hz}, \operatorname{ArH}), 7.5(1 \mathrm{H}, \mathrm{t}, J=7.8 \mathrm{~Hz}, \operatorname{ArH})$, $7.3(1 \mathrm{H}, \mathrm{t}, J=7.8 \mathrm{~Hz}, \mathrm{ArH}), 3.6\left(2 \mathrm{H}, \mathrm{t}, J=7.1 \mathrm{~Hz}, \mathrm{NHCH}_{2}\right), 3.2\left(2 \mathrm{H}, \mathrm{t}, J=7.1 \mathrm{~Hz}, \mathrm{CH}_{2}\right), 3.0(4 \mathrm{H}, \mathrm{t}$, 
$\left.J=7.8 \mathrm{~Hz}, \mathrm{CH}_{2} \mathrm{NH}_{2}\right), 2.6\left(2 \mathrm{H}, \mathrm{t}, J=6.8 \mathrm{~Hz}, \mathrm{CH}_{2}\right), 2.1\left(2 \mathrm{H}, \mathrm{p}, J=7.6,7.3 \mathrm{~Hz}, \mathrm{CH}_{2}\right), 1.6-1.7(2 \mathrm{H}, \mathrm{m}$, $\left.\mathrm{CH}_{2}\right)$, 1.3-1.5 (6H, br, $\left.\mathrm{CH}_{2}\right)$; IR (film) $v\left(\mathrm{~cm}^{-1}\right)$ : 1567.5, 2855.0, 2928.4, 3350.4; MS (FAB) $m / z(\mathrm{M}+1) 284.3,197.0,185.0$; MS-HR (FAB) Calc. for $\mathrm{C}_{18} \mathrm{H}_{25} \mathrm{~N}_{3}: 283.2048$ Found: 283.20426.

\subsection{9. $N^{\prime}$-(2,3-Dihydro-1H-cyclopenta[b]quinolin-9-yl)heptane-1,7-diamine (4f)}

A mixture of 3 (0.75 g, $3.5 \mathrm{mmol})$, 1,7-diaminoheptane (0.92 g, $7 \mathrm{mmol})$, phenol (1.5 g), and NaI $(0.07 \mathrm{~g})$ was combined as above to afford $\mathbf{4 f}$ as an oil. Compound $\mathbf{4 f}$ : yield $72 \% ;{ }^{1} \mathrm{H}$ NMR $\left(\mathrm{CDCl}_{3}\right)$ ( $\delta$ ppm.): $7.9(1 \mathrm{H}, \mathrm{d}, J=7.5 \mathrm{~Hz}, \mathrm{ArH}), 7.7(1 \mathrm{H}, \mathrm{t}, J=8.3 \mathrm{~Hz}, \mathrm{ArH}), 7.5(1 \mathrm{H}, \mathrm{t}, J=7.1 \mathrm{~Hz}, \mathrm{ArH}), 7.4$ $(1 \mathrm{H}, \mathrm{t}, J=6.9 \mathrm{~Hz}, \mathrm{ArH}), 4.6(1 \mathrm{H}, \mathrm{s}, \mathrm{NH}), 3.6\left(2 \mathrm{H}, \mathrm{m}, \mathrm{NHCH}_{2}\right), 3.3\left(2 \mathrm{H}, \mathrm{t}, J=7.5 \mathrm{~Hz}, \mathrm{CH}_{2}\right), 3.0(2 \mathrm{H}, \mathrm{t}$, $\left.J=7.7 \mathrm{~Hz}, \mathrm{CH}_{2} \mathrm{NH}_{2}\right), 2.6\left(2 \mathrm{H}, \mathrm{t}, J=6.7 \mathrm{~Hz}, \mathrm{CH}_{2}\right), 2.1\left(2 \mathrm{H}, \mathrm{p}, J=7.7,7.3 \mathrm{~Hz}, \mathrm{CH}_{2}\right), 1.7\left(2 \mathrm{H}, \mathrm{s}, \mathrm{NH}_{2}\right)$, 1.5-1.6 (2H, m, $\left.\mathrm{CH}_{2} \mathrm{CH}_{2}\right), 1.2-1.5\left(8 \mathrm{H}, \mathrm{br}, \mathrm{CH}_{2} \mathrm{CH}_{2}\right)$; IR (film) v ( $\left.\mathrm{cm}^{-1}\right)$ : 1567.9, 2853.9, 2927.9, 3294.4; MS (FAB) $m / z(M+1)$ 298.3, 197.0, 185.0; MS-HR (FAB) Calc. for $\mathrm{C}_{19} \mathrm{H}_{27} \mathrm{~N}_{3}: 297.2205$ Found: $298.22911(\mathrm{M}+1)$.

\subsubsection{0. $N^{\prime}$-(2,3-Dihydro-1H-cyclopenta[ $\left.b\right]$ quinolin-9-yl)octane-1,8-diamine (4g)}

A mixture of $3(0.71 \mathrm{~g}, 3.5 \mathrm{mmol})$, 1,8-diaminooctane (1.00 g, $7 \mathrm{mmol})$, phenol (1.5 g), and NaI $(0.07 \mathrm{~g})$ was combined as above to afford $\mathbf{4 g}$ as an oil. Compound $4 \mathrm{~g}$ : yield $68 \%$; ${ }^{1} \mathrm{H} \mathrm{NMR}\left(\mathrm{CDCl}_{3}\right)$ ( $\delta$ ppm.): $7.8(1 \mathrm{H}, \mathrm{d}, J=8.3 \mathrm{~Hz}, \mathrm{ArH}), 7.5(1 \mathrm{H}, \mathrm{t}, J=7.3 \mathrm{~Hz}, \mathrm{ArH}), 7.3(1 \mathrm{H}, \mathrm{t}, J=7.5 \mathrm{~Hz}, \mathrm{ArH}), 4.5$ $(1 \mathrm{H}, \mathrm{s}, \mathrm{NH}), 3.5\left(2 \mathrm{H}, \mathrm{m}, \mathrm{NHCH}_{2}\right), 3.1\left(2 \mathrm{H}, \mathrm{t}, J=7.1 \mathrm{~Hz}, \mathrm{CH}_{2}\right), 3.0\left(2 \mathrm{H}, \mathrm{d}, J=7.7 \mathrm{~Hz}, \mathrm{CH}_{2} \mathrm{NH}_{2}\right), 2.6$ $\left(2 \mathrm{H}, \mathrm{t}, J=6.5 \mathrm{~Hz}, \mathrm{CH}_{2}\right), 1.5-1.6\left(4 \mathrm{H}, \mathrm{br}, \mathrm{CH}_{2}, \mathrm{NH}_{2}\right), 1.1-1.5\left(10 \mathrm{H}, \mathrm{br}, \mathrm{CH}_{2}\right)$; IR (film) $v\left(\mathrm{~cm}^{-1}\right)$ : 1567.2, 2853.2, 2925.5, 3329.5; MS (FAB) $m / z(\mathrm{M}+1) 312.5$, 197.1, 185.1; MS-HR (FAB) Calcd. for $\mathrm{C}_{20} \mathrm{H}_{29} \mathrm{~N}_{3}$ : 311.2361 Found: $312.24442(\mathrm{M}+1)$.

\subsubsection{1. $N^{\prime}$-(2,3-Dihydro-1H-cyclopenta[b]quinolin-9-yl)nonane-1,9-diamine (4f)}

A mixture of 3 (0.71 g, $3.5 \mathrm{mmol})$, 1,9-diaminononane (1.10 g, $7 \mathrm{mmol})$, phenol (1.5 g), and NaI $(0.07 \mathrm{~g})$ was combined as above to afford $\mathbf{4 f}$ as an oil. Compound $\mathbf{4 f}$ : yield $66 \% ;{ }^{1} \mathrm{H} \mathrm{NMR}\left(\mathrm{CDCl}_{3}\right)$ ( $\delta$ ppm.): $7.8(1 \mathrm{H}, \mathrm{d}, J=7.3 \mathrm{~Hz}, \mathrm{ArH}), 7.6(1 \mathrm{H}, \mathrm{d}, J=7.5 \mathrm{~Hz}, \mathrm{ArH}), 7.5$ (1H, t, $J=6.9 \mathrm{~Hz}, \mathrm{ArH}), 7.2$ $(1 \mathrm{H}, \mathrm{t}, J=6.9 \mathrm{~Hz}, \mathrm{ArH}), 4.6(1 \mathrm{H}, \mathrm{s}, \mathrm{NH}), 3.5\left(2 \mathrm{H}, \mathrm{m}, \mathrm{NHCH}_{2}\right), 3.1\left(2 \mathrm{H}, \mathrm{t}, J=7.1 \mathrm{~Hz} \mathrm{CH}_{2}\right), 3.0(2 \mathrm{H}, \mathrm{t}$, $\left.J=7.7 \mathrm{~Hz}, \mathrm{CH}_{2} \mathrm{NH}_{2}\right), 2.6\left(2 \mathrm{H}, \mathrm{t}, J=6.9 \mathrm{~Hz} \mathrm{CH}_{2}\right), 2.1\left(2 \mathrm{H}, \mathrm{p}, J=7.7,7.7 \mathrm{~Hz} \mathrm{CH}_{2}\right), 1,7\left(2 \mathrm{H}, \mathrm{s}, \mathrm{NH}_{2}\right)$, 1.5-1.6 (2H, m, $\left.\mathrm{CH}_{2} \mathrm{CH}_{2}\right), 1.1-1.3\left(14 \mathrm{H}, \mathrm{br}, \mathrm{CH}_{2} \mathrm{CH}_{2}\right)$; IR (film) $v\left(\mathrm{~cm}^{-1}\right)$ : 1568.0, 2852.5, 2925.7, 3294.4; MS (FAB) $m / z(M+1) 326.3$, 185.0; MS-HR (FAB) Calc. for $\mathrm{C}_{21} \mathrm{H}_{31} \mathrm{~N}_{3}$ : 325.2518 Found: $326.26086(\mathrm{M}+1)$.

\subsubsection{2. $N$-\{5-[2-(2,3-Dihydro-1H-cyclopenta[b]quinolin-9-ylamino)ethylcarbamoyl]pyridin-2-yl $\}$} hydrazinecarboxylic Acid tert-Butyl Ester (5a)

To the 2-chloro-4,6-dimethoxy-1,3,5-triazine (CDMT) (1.76 g, $10 \mathrm{mmol})$ and 2 (2.53 g, $10 \mathrm{mmol})$ in THF $(10 \mathrm{~mL}), N$-methylmorpholine $(1.1 \mathrm{~mL}, 10 \mathrm{mmol})$ was added drop by drop at a rate sufficient to keep the temperature between $-5{ }^{\circ} \mathrm{C}$ and $0{ }^{\circ} \mathrm{C}$. Stirring was continued at $0{ }^{\circ} \mathrm{C}$ for $1-4 \mathrm{~h}$ until all CDMT was consumed. Subsequently, to the crude mixture obtained as described above, $4 \mathbf{a}(2.27 \mathrm{~g}$, $10 \mathrm{mmol})$ in THF $(8 \mathrm{~mL})$ at $-5{ }^{\circ} \mathrm{C}$ to $0{ }^{\circ} \mathrm{C}$ was added. Stirring was continued at $0{ }^{\circ} \mathrm{C}$ for $2 \mathrm{~h}$, and then 
for $12 \mathrm{~h}$ at room temperature. Precipitate formed and was isolated by filtration. Recrystallization from ethyl acetate afforded the desired product 5a as a yellow solid. Compound 5a: yield 68\%; mp 200-202 ${ }^{\circ} \mathrm{C}$; ${ }^{1} \mathrm{H}$ NMR $\left(\mathrm{CD}_{3} \mathrm{OD}\right)(\delta \mathrm{ppm}$.): $8.5(1 \mathrm{H}, \mathrm{s}, \mathrm{ArH}), 8.3(1 \mathrm{H}, \mathrm{d}, J=8.3 \mathrm{~Hz}, \mathrm{ArH}), 7.9(1 \mathrm{H}, \mathrm{d}$, $J=11.5 \mathrm{~Hz}, \mathrm{ArH}), 7.8(1 \mathrm{H}, \mathrm{t}, J=7.1 \mathrm{~Hz}, \mathrm{ArH}), 7.7(1 \mathrm{H}, \mathrm{d}, J=8.5 \mathrm{~Hz}, \mathrm{ArH}), 7.6(1 \mathrm{H}, \mathrm{t}, J=7.1 \mathrm{~Hz}$, $\operatorname{ArH}), 6.7(1 \mathrm{H}, \mathrm{d}, J=8.5 \mathrm{~Hz}, \mathrm{ArH}), 4.0\left(2 \mathrm{H}, \mathrm{t}, J=5.6 \mathrm{~Hz}, \mathrm{CH}_{2}\right), 3.7\left(2 \mathrm{H}, \mathrm{t}, J=6.0 \mathrm{~Hz}, \mathrm{CH}_{2}\right), 3.4(2 \mathrm{H}$, t, $\left.J=6.6 \mathrm{~Hz}, \mathrm{CH}_{2}\right), 3.1\left(2 \mathrm{H}, \mathrm{t}, J=7.8 \mathrm{~Hz}, \mathrm{CH}_{2}\right), 2.3\left(2 \mathrm{H}, \mathrm{p}, J=7.6,7.8 \mathrm{~Hz}, \mathrm{CH}_{2}\right), 1.3-1.5(9 \mathrm{H}, \mathrm{m}$, BOC); IR (KBr) $v\left(\mathrm{~cm}^{-1}\right): 1464.2,1610.9,1740.6,2869.2,2936.2,3422.1 ; \mathrm{MS}(\mathrm{FAB}) \mathrm{m} / z(\mathrm{M}+1)$ 463.4, 363.2; 183.1; MS-HR (FAB) Calc. for $\mathrm{C}_{25} \mathrm{H}_{30} \mathrm{~N}_{6} \mathrm{O}_{3}$ : 462.23794 Found: $463.24583(\mathrm{M}+1)$.

\subsubsection{3. $N$-\{5-[3-(2,3-Dihydro-1H-cyclopenta[b]quinolin-9-ylamino)propylcarbamoyl]pyridin-2-yl $\}$} hydrazinecarboxylic Acid tert-Butyl Ester (5b)

A mixture of 2-chloro-4,6-dimethoxy-1,3,5-triazine (CDMT) (1.76 g, $10 \mathrm{mmol}), 2$ (2.53 g, $10 \mathrm{mmol})$ in THF $(10 \mathrm{~mL})$, and $N$-methylmorpholine $(1.1 \mathrm{~mL}, 10 \mathrm{mmol})$ and after $4 \mathrm{~h} 4 \mathbf{b}(2.41 \mathrm{~g}, 10 \mathrm{mmol})$ in THF were combined as above to afford $\mathbf{5 b}$ as yellow solid. Compound $\mathbf{5 b}$ : yield $66 \%$; $\mathrm{mp} 159-161{ }^{\circ} \mathrm{C}$; ${ }^{1} \mathrm{H}$ NMR $\left(\mathrm{CD}_{3} \mathrm{OD}\right)(\delta \mathrm{ppm}):. 8.5(1 \mathrm{H}, \mathrm{s}, \mathrm{ArH}), 8.3(1 \mathrm{H}, \mathrm{d}, J=8.5 \mathrm{~Hz}, \mathrm{ArH}), 7.9(1 \mathrm{H}, \mathrm{d}, J=9.0 \mathrm{~Hz}$, $\operatorname{ArH}), 7.8(1 \mathrm{H}, \mathrm{t}, J=5.9 \mathrm{~Hz}, \mathrm{ArH}), 7.7(1 \mathrm{H}, \mathrm{d}, J=7.6 \mathrm{~Hz}, \mathrm{ArH}), 7.6(1 \mathrm{H}, \mathrm{t}, J=8.5 \mathrm{~Hz}, \mathrm{ArH}), 6.7(1 \mathrm{H}$, $\mathrm{d}, J=8.5 \mathrm{~Hz}, \mathrm{ArH}), 3.8\left(2 \mathrm{H}, \mathrm{t}, J=6.8 \mathrm{~Hz}, \mathrm{CH}_{2}\right), 3.7\left(2 \mathrm{H}, \mathrm{t}, J=4.6 \mathrm{~Hz}, \mathrm{CH}_{2}\right), 3.5(2 \mathrm{H}, \mathrm{t}, J=6.4 \mathrm{~Hz}$, $\left.\mathrm{CH}_{2}\right), 3.3(2 \mathrm{H}, \mathrm{m}, \mathrm{NH}), 3.1\left(2 \mathrm{H}, \mathrm{t}, J=7.8 \mathrm{~Hz}, \mathrm{CH}_{2}\right), 2.5(2 \mathrm{H}, \mathrm{m}, \mathrm{NH}), 2.2(2 \mathrm{H}, \mathrm{p}, J=7.8,7.6 \mathrm{~Hz}$, $\left.\mathrm{CH}_{2}\right), 2.0\left(2 \mathrm{H}, \mathrm{p}, J=7.6,5.1 \mathrm{~Hz}, \mathrm{CH}_{2}\right), 1.3-1.5(9 \mathrm{H}, \mathrm{m}, \mathrm{BOC}) ; \mathrm{IR}(\mathrm{KBr}) v\left(\mathrm{~cm}^{-1}\right): 1470.7,1635.8$, 1721.1, 2853.4, 2967.2, 3259.6; MS (FAB) $m / z(M+1)$ 477.3, 377.3, 183.0; MS-HR (FAB) Calc. for $\mathrm{C}_{26} \mathrm{H}_{32} \mathrm{~N}_{6} \mathrm{O}_{3}: 476.25359$ Found: $477.25943(\mathrm{M}+1)$.

3.1.14. $N$ - $\{5$-[4-(2,3-Dihydro-1H-cyclopenta[b]quinolin-9-ylamino)butylcarbamoyl]pyridin-2-yl $\}$ hydrazinecarboxylic Acid tert-Butyl Ester (5c)

A mixture of 2-chloro-4,6-dimethoxy-1,3,5-triazine (CDMT) (1.76 g, $10 \mathrm{mmol}), 2$ (2.53 g, $10 \mathrm{mmol})$ in THF $(10 \mathrm{~mL})$, and $N$-methylmorpholine $(1.1 \mathrm{~mL}, 10 \mathrm{mmol})$ and after $4 \mathrm{~h} 4 \mathrm{c}(2.55 \mathrm{~g}, 10 \mathrm{mmol})$ in THF were combined as above to afford $\mathbf{5 c}$ as yellow solid. Compound $\mathbf{5 c}$ : yield $67 \%$; $\mathrm{mp} 145-148{ }^{\circ} \mathrm{C}$; ${ }^{1} \mathrm{H}$ NMR $\left(\mathrm{CD}_{3} \mathrm{OD}\right)(\delta$ ppm.): $8.5(1 \mathrm{H}, \mathrm{s}, \mathrm{ArH}), 8.3(1 \mathrm{H}, \mathrm{d}, J=7.9 \mathrm{~Hz}, \mathrm{ArH}), 7.9(1 \mathrm{H}, \mathrm{m}, \mathrm{ArH}), 7.8(1 \mathrm{H}$, t, $J=7.3 \mathrm{~Hz}, \mathrm{ArH}), 7.7(1 \mathrm{H}, \mathrm{d}, J=6.9 \mathrm{~Hz}, \mathrm{ArH}), 7.6(1 \mathrm{H}, \mathrm{t}, J=6.9 \mathrm{~Hz}, \mathrm{ArH}), 6.7(1 \mathrm{H}, \mathrm{d}, J=8.3 \mathrm{~Hz}$, $\mathrm{ArH}), 3.8\left(2 \mathrm{H}, \mathrm{t}, J=6.9 \mathrm{~Hz}, \mathrm{CH}_{2}\right), 3.7\left(2 \mathrm{H}, \mathrm{t}, J=4.6 \mathrm{~Hz}, \mathrm{CH}_{2}\right), 3.3-3.4\left(4 \mathrm{H}, \mathrm{m}, \mathrm{NH}, \mathrm{CH}_{2}\right), 3.1(2 \mathrm{H}, \mathrm{t}$, $\left.J=7.9 \mathrm{~Hz}, \mathrm{CH}_{2}\right), 2.5(2 \mathrm{H}, \mathrm{m}, \mathrm{NH}), 2.3\left(2 \mathrm{H}, \mathrm{p}, J=7.7,9.3 \mathrm{~Hz}, \mathrm{CH}_{2}\right), 1.7\left(4 \mathrm{H}, \mathrm{m}, \mathrm{CH}_{2}\right), 1.3-1.5(9 \mathrm{H}$, $\mathrm{m}, \mathrm{BOC})$; IR (KBr) $v\left(\mathrm{~cm}^{-1}\right)$ : 1466.6, 1634.1, 1721.1, 2934.4, 3260.6; MS (FAB) $\mathrm{m} / z(\mathrm{M}+1) 491.3$, 391.3, 185.1; MS-HR (FAB) Calc. for $\mathrm{C}_{27} \mathrm{H}_{34} \mathrm{~N}_{6} \mathrm{O}_{3}$ : 490.26924 Found: $491.27811(\mathrm{M}+1)$.

\subsubsection{5. $N$ - $\{5$-[5-(2,3-Dihydro-1H-cyclopenta[b]quinolin-9-ylamino)pentylcarbamoyl]pyridin-2-yl $\}$} hydrazinecarboxylic Acid tert-Butyl Ester (5d)

A mixture of 2-chloro-4,6-dimethoxy-1,3,5-triazine (CDMT) (1.76 g, $10 \mathrm{mmol}), 2$ (2.53 g, $10 \mathrm{mmol})$ in THF $(10 \mathrm{~mL})$, and $N$-methylmorpholine $(1.1 \mathrm{~mL}, 10 \mathrm{mmol})$ and after $4 \mathrm{~h} \mathrm{4d}(2.69 \mathrm{~g}, 10 \mathrm{mmol})$ in THF were combined as above to afford $\mathbf{5 d}$ as yellow solid. Compound $\mathbf{5 d}$ : yield $70 \%$; mp 106-108 ${ }^{\circ} \mathrm{C}$; ${ }^{1} \mathrm{H}$ NMR $\left(\mathrm{CD}_{3} \mathrm{OD}\right)(\delta \mathrm{ppm}):. 8.4(1 \mathrm{H}, \mathrm{s}, \mathrm{ArH}), 8.2(1 \mathrm{H}, \mathrm{d}, J=8.7 \mathrm{~Hz}, \mathrm{ArH}), 7.9(1 \mathrm{H}, \mathrm{d}, J=8.9 \mathrm{~Hz}$, 
ArH), $7.8(1 \mathrm{H}, \mathrm{t}, J=5.6 \mathrm{~Hz}, \mathrm{ArH}), 7.7(1 \mathrm{H}, \mathrm{d}, J=8.1 \mathrm{~Hz}, \mathrm{ArH}), 7.5(1 \mathrm{H}, \mathrm{t}, J=8.3 \mathrm{~Hz}, \mathrm{ArH}), 6.6(1 \mathrm{H}$, $\mathrm{d}, J=8.9 \mathrm{~Hz}, \mathrm{ArH}), 3.8\left(2 \mathrm{H}, \mathrm{t}, J=6.9 \mathrm{~Hz}, \mathrm{CH}_{2}\right), 3.7\left(2 \mathrm{H}, \mathrm{t}, J=4.8 \mathrm{~Hz}, \mathrm{CH}_{2}\right), 3.4(2 \mathrm{H}, \mathrm{m}, \mathrm{NH}), 3.3$ $\left(2 \mathrm{H}, \mathrm{t}, J=7.1 \mathrm{~Hz}, \mathrm{CH}_{2}\right), 3.1\left(2 \mathrm{H}, \mathrm{t}, J=7.7 \mathrm{~Hz}, \mathrm{CH}_{2}\right), 2.5(2 \mathrm{H}, \mathrm{m}, \mathrm{NH}), 2.2(2 \mathrm{H}, \mathrm{p}, J=7.9,7.5 \mathrm{~Hz}$, $\left.\mathrm{CH}_{2}\right)$, 1.6-1.8 (6H, m, $\left.\mathrm{CH}_{2}\right), 1.3-1.5(9 \mathrm{H}, \mathrm{m}, \mathrm{BOC})$; IR $(\mathrm{KBr}) \vee\left(\mathrm{cm}^{-1}\right)$ : 1465.2, 1634.4, 1716.0, 2855.0, 2927.0, 3275.6; MS (FAB) $m / z(\mathrm{M}+1)$ 505.4, 405.3, 185.0; MS-HR (FAB) Calc. for $\mathrm{C}_{28} \mathrm{H}_{36} \mathrm{~N}_{6} \mathrm{O}_{3}$ : 504.28489 Found: 505.29257 (M+1).

3.1.16. $N$-\{5-[6-(2,3-Dihydro-1H-cyclopenta[b]quinolin-9-ylamino)hexylcarbamoyl]pyridin-2-yl $\}$ hydrazinecarboxylic Acid tert-Butyl Ester (5e)

A mixture of 2-chloro-4,6-dimethoxy-1,3,5-triazine (CDMT) (1.76 g, $10 \mathrm{mmol}), 2$ (2.53 g, $10 \mathrm{mmol})$ in THF $(10 \mathrm{~mL})$, and $N$-methylmorpholine $(1.1 \mathrm{~mL}, 10 \mathrm{mmol})$ and after $4 \mathrm{~h} 4 \mathrm{c}(2.83 \mathrm{~g}, 10 \mathrm{mmol})$ in THF were combined as above to afford $\mathbf{5 c}$ as yellow solid. Compound $\mathbf{5 c}$ : yield $75 \%$; $\mathrm{mp} 110-112{ }^{\circ} \mathrm{C}$; ${ }^{1} \mathrm{H}$ NMR $\left(\mathrm{CD}_{3} \mathrm{OD}\right)(\delta \mathrm{ppm}):. 8.5(1 \mathrm{H}, \mathrm{s}, \mathrm{ArH}), 8.3(1 \mathrm{H}, \mathrm{d}, J=8.1 \mathrm{~Hz}, \mathrm{ArH}), 8.0(1 \mathrm{H}, \mathrm{d}, J=9.1 \mathrm{~Hz}$, $\operatorname{ArH}), 7.8(1 \mathrm{H}, \mathrm{t}, J=5.7 \mathrm{~Hz}, \mathrm{ArH}), 7.7(1 \mathrm{H}, \mathrm{d}, J=7.5 \mathrm{~Hz}, \mathrm{ArH}), 7.6(1 \mathrm{H}, \mathrm{t}, J=7.1 \mathrm{~Hz}, \mathrm{ArH}), 6.7(1 \mathrm{H}$, $\mathrm{d}, J=8.9 \mathrm{~Hz}, \mathrm{ArH}), 3.8\left(2 \mathrm{H}, \mathrm{t}, J=7.2 \mathrm{~Hz}, \mathrm{CH}_{2}\right), 3.7\left(2 \mathrm{H}, \mathrm{t}, J=4.8 \mathrm{~Hz}, \mathrm{CH}_{2}\right), 3.4\left(4 \mathrm{H}, \mathrm{m}, \mathrm{NH}, \mathrm{CH}_{2}\right)$, $3.2\left(2 \mathrm{H}, \mathrm{t}, J=5.4 \mathrm{~Hz}, \mathrm{CH}_{2}\right), 2.5(2 \mathrm{H}, \mathrm{m}, \mathrm{NH}), 2.3\left(2 \mathrm{H}, \mathrm{p}, J=7.7,7.7 \mathrm{~Hz}, \mathrm{CH}_{2}\right), 1.8\left(4 \mathrm{H}, \mathrm{m}, \mathrm{CH}_{2}\right), 1.7$ $\left(4 \mathrm{H}, \mathrm{m}, \mathrm{CH}_{2}\right), 1.3-1.5(9 \mathrm{H}, \mathrm{m}, \mathrm{BOC})$; IR $(\mathrm{KBr}) \vee\left(\mathrm{cm}^{-1}\right): 1466.2,1634.4,1717.01,2855.5,2930.2$, 3250.3; MS (FAB) $m / z(\mathrm{M}+1) 519.3,419.3,185.0$; MS-HR (FAB) Calc. for $\mathrm{C}_{29} \mathrm{H}_{38} \mathrm{~N}_{6} \mathrm{O}_{3}: 518.30054$ Found: $519.30705(\mathrm{M}+1)$.

3.1.17. $N$ - $\{5$-[7-(2,3-Dihydro-1H-cyclopenta[b]quinolin-9-ylamino)heptylcarbamoyl]pyridin-2-yl $\}$ hydrazinecarboxylic Acid tert-Butyl Ester (5f)

A mixture of 2-chloro-4,6-dimethoxy-1,3,5-triazine (CDMT) (1.76 g, $10 \mathrm{mmol}), 2$ (2.53 g, $10 \mathrm{mmol})$ in THF $(10 \mathrm{~mL})$, and $N$-methylmorpholine $(1.1 \mathrm{~mL}, 10 \mathrm{mmol})$ and after $4 \mathrm{~h} 4 \mathbf{d}(2.97 \mathrm{~g}, 10 \mathrm{mmol})$ in THF were combined as above to afford $\mathbf{5 d}$ as yellow solid. Compound $\mathbf{5 d}$ : yield $66 \%$; $\mathrm{mp} 105-107{ }^{\circ} \mathrm{C}$; ${ }^{1} \mathrm{H}$ NMR $\left(\mathrm{CD}_{3} \mathrm{OD}\right)(\delta \mathrm{ppm}):. 8.5(1 \mathrm{H}, \mathrm{s}, \mathrm{ArH}), 8.3(1 \mathrm{H}, \mathrm{d}, J=8.7 \mathrm{~Hz}, \mathrm{ArH}), 8.0(1 \mathrm{H}, \mathrm{d}, J=6.9 \mathrm{~Hz}$, $\operatorname{ArH}), 7.8(1 \mathrm{H}, \mathrm{t}, J=8.1 \mathrm{~Hz}, \mathrm{ArH}), 7.7(1 \mathrm{H}, \mathrm{d}, J=8.5 \mathrm{~Hz}, \mathrm{ArH}), 7.6(1 \mathrm{H}, \mathrm{t}, J=6.9 \mathrm{~Hz}, \mathrm{ArH}), 6.7(1 \mathrm{H}$, $\mathrm{d}, J=8.7 \mathrm{~Hz}, \mathrm{ArH}), 4.0\left(2 \mathrm{H}, \mathrm{t}, J=4.0 \mathrm{~Hz}, \mathrm{CH}_{2}\right), 3.8\left(2 \mathrm{H}, \mathrm{t}, J=7.1 \mathrm{~Hz}, \mathrm{CH}_{2}\right), 3.4(2 \mathrm{H}, \mathrm{t}, J=6.1 \mathrm{~Hz}$, $\left.\mathrm{CH}_{2}\right), 3.2\left(2 \mathrm{H}, \mathrm{t}, J=7.9 \mathrm{~Hz}, \mathrm{CH}_{2}\right), 2.3\left(2 \mathrm{H}, \mathrm{p}, J=8.1,6.9 \mathrm{~Hz}, \mathrm{CH}_{2}\right), 1.8\left(4 \mathrm{H}, \mathrm{m}, \mathrm{CH}_{2}\right), 1.6(6 \mathrm{H}, \mathrm{m}$, $\left.\mathrm{CH}_{2}\right), 1.3-1.5(9 \mathrm{H}, \mathrm{m}, \mathrm{BOC})$; IR $(\mathrm{KBr}) \vee\left(\mathrm{cm}^{-1}\right)$ : 1566.5, 1717.7, 2855.1, 2927.2, 3295.0; MS (FAB) $m / z(\mathrm{M}+1)$ 533.3, 433.3, 185.0; MS-HR (FAB) Calc. for $\mathrm{C}_{30} \mathrm{H}_{40} \mathrm{~N}_{6} \mathrm{O}_{3}: 532.31619$ Found: $533.32329(\mathrm{M}+1)$.

3.1.18. $N$-\{5-[8-(2,3-Dihydro-1H-cyclopenta[b]quinolin-9-ylamino)octylcarbamoyl]pyridin-2-yl $\}$ hydrazinecarboxylic Acid tert-Butyl Ester (5g)

A mixture of 2-chloro-4,6-dimethoxy-1,3,5-triazine (CDMT) (1.76 g, $10 \mathrm{mmol}), 2$ (2.53 g, $10 \mathrm{mmol})$ in THF $(10 \mathrm{~mL})$, and $N$-methylmorpholine $(1.1 \mathrm{~mL}, 10 \mathrm{mmol})$ and after $4 \mathrm{~h} 4 \mathrm{c}(3.11 \mathrm{~g}, 10 \mathrm{mmol})$ in THF were combined as above to afford $\mathbf{5 c}$ as yellow solid. Compound $\mathbf{5 c}$ : yield $46 \%$; $\mathrm{mp} 134-136{ }^{\circ} \mathrm{C}$; ${ }^{1} \mathrm{H}$ NMR $\left(\mathrm{CD}_{3} \mathrm{OD}\right)(\delta \mathrm{ppm}):. 8.5(1 \mathrm{H}, \mathrm{s}, \mathrm{ArH}), 8.3(1 \mathrm{H}, \mathrm{d}, J=8.7 \mathrm{~Hz}, \mathrm{ArH}), 8.0(1 \mathrm{H}, \mathrm{d}, J=6.6 \mathrm{~Hz}$, $\operatorname{ArH}), 7.8(1 \mathrm{H}, \mathrm{t}, J=6.9 \mathrm{~Hz}, \mathrm{ArH}), 7.7(1 \mathrm{H}, \mathrm{d}, J=8.5 \mathrm{~Hz}, \mathrm{ArH}), 7.6(1 \mathrm{H}, \mathrm{t}, J=6.9 \mathrm{~Hz}, \operatorname{ArH}), 6.7(1 \mathrm{H}$, 
$\mathrm{d}, J=8.7 \mathrm{~Hz}, \mathrm{ArH}), 4.6(2 \mathrm{H}, \mathrm{m}, \mathrm{NH}), 4.0\left(2 \mathrm{H}, \mathrm{t}, J=3.8 \mathrm{~Hz}, \mathrm{CH}_{2}\right), 3.8\left(2 \mathrm{H}, \mathrm{t}, J=7.3 \mathrm{~Hz}, \mathrm{CH}_{2}\right), 3.4$ $\left(4 \mathrm{H}, \mathrm{m}, \mathrm{NH}, \mathrm{CH}_{2}\right), 3.2\left(2 \mathrm{H}, \mathrm{t}, J=7.9 \mathrm{~Hz}, \mathrm{CH}_{2}\right), 2.3\left(2 \mathrm{H}, \mathrm{p}, J=7.7,6.9 \mathrm{~Hz}, \mathrm{CH}_{2}\right), 1.7\left(6 \mathrm{H}, \mathrm{m}, \mathrm{CH}_{2}\right), 1.6$ $\left(6 \mathrm{H}, \mathrm{m}, \mathrm{CH}_{2}\right), 1.3-1.5(9 \mathrm{H}, \mathrm{m}, \mathrm{BOC})$; IR $(\mathrm{KBr}) v\left(\mathrm{~cm}^{-1}\right): 1466.7,1634.1,1717.2,2854.3,2928.2$, 3259.6; MS (FAB) $m / z(\mathrm{M}+1)$ 547.3, 447.3, 183.0; MS-HR (FAB) Calc. for $\mathrm{C}_{31} \mathrm{H}_{42} \mathrm{~N}_{6} \mathrm{O}_{3}: 546.33184$ Found: $547.33977(\mathrm{M}+1)$.

3.1.19. $N$ - $\{5$-[9-(2,3-Dihydro-1H-cyclopenta[b]quinolin-9-ylamino)nonylcarbamoyl]pyridin-2-yl $\}$ hydrazinecarboxylic Acid tert-Butyl Ester (5h)

A mixture of 2-chloro-4,6-dimethoxy-1,3,5-triazine (CDMT) (1.76 g, $10 \mathrm{mmol}), 2$ (2.53 g, $10 \mathrm{mmol})$ in THF $(10 \mathrm{~mL})$, and $N$-methylmorpholine $(1.1 \mathrm{~mL}, 10 \mathrm{mmol})$ and after $4 \mathrm{~h} 4 \mathbf{d}(3.26 \mathrm{~g}, 10 \mathrm{mmol})$ in THF were combined as above to afford $\mathbf{5 d}$ as yellow solid. Compound $\mathbf{5 d}$ : yield $62 \%$; mp $81-83{ }^{\circ} \mathrm{C}$; ${ }^{1} \mathrm{H}$ NMR $\left(\mathrm{CD}_{3} \mathrm{OD}\right)(\delta$ ppm.): $8.5(1 \mathrm{H}, \mathrm{s}, \mathrm{ArH}), 8.4(1 \mathrm{H}, \mathrm{d}, J=8.3 \mathrm{~Hz}, \mathrm{ArH}), 8.0(1 \mathrm{H}, \mathrm{m}, \mathrm{ArH}), 7.8$ $(1 \mathrm{H}, \mathrm{t}, J=8.3 \mathrm{~Hz}, \mathrm{ArH}), 7.7(1 \mathrm{H}, \mathrm{d}, J=8.1 \mathrm{~Hz}, \mathrm{ArH}), 7.5(1 \mathrm{H}, \mathrm{t}, J=7.3 \mathrm{~Hz}, \mathrm{ArH}), 6.6(1 \mathrm{H}, \mathrm{d}, J=9.1 \mathrm{~Hz}$, ArH), $4.6(2 \mathrm{H}, \mathrm{m}, \mathrm{NH}), 4.0\left(2 \mathrm{H}, \mathrm{m}, \mathrm{CH}_{2}\right), 3.8\left(2 \mathrm{H}, \mathrm{m}, \mathrm{CH}_{2}\right), 3.4(2 \mathrm{H}, \mathrm{m}, \mathrm{NH}), 3.0\left(2 \mathrm{H}, \mathrm{m}, \mathrm{CH}_{2}\right), 2.7$ $\left(2 \mathrm{H}, \mathrm{m}, \mathrm{CH}_{2}\right), 2.0\left(2 \mathrm{H}, \mathrm{p}, J=7.7,6.9 \mathrm{~Hz}, \mathrm{CH}_{2}\right), 1.8\left(6 \mathrm{H}, \mathrm{m}, \mathrm{CH}_{2}\right), 1.7\left(8 \mathrm{H}, \mathrm{m}, \mathrm{CH}_{2}\right), 1.3-1.5(9 \mathrm{H}, \mathrm{m}$, BOC); IR (KBr) $v\left(\mathrm{~cm}^{-1}\right): 1489.8,1636.3,1709.1,2868.7,2927.8,3421.7$; MS (FAB) $\mathrm{m} / z(\mathrm{M}+1)$ 561.5, 461.3, 183.0; MS-HR (FAB) Calc. for $\mathrm{C}_{32} \mathrm{H}_{44} \mathrm{~N}_{6} \mathrm{O}_{3}: 560.34749$ Found: $561.35526(\mathrm{M}+1)$.

3.1.20. 6-Hydrazino- $N$-[2-(2,3-dihydro- $1 H$-cyclopenta[ $b]$ quinolin-9-ylamino)Ethyl]nicotinamide hydrochloride (6a)

Compound 5a (0.20 g, $0.43 \mathrm{mmol})$ was dissolved in ether $(2 \mathrm{~mL}), \mathrm{HCl} / \mathrm{ether}(4 \mathrm{~mL})$ was added, and the reaction mixture was stirred at room temperature. After $24 \mathrm{~h}$, the solution became cloudy and precipitate formed. The precipitate was isolated by filtration and the solid was washed with ether and dried. Compound 6a: yield 34\%; mp $235-136{ }^{\circ} \mathrm{C} ;{ }^{1} \mathrm{H}$ NMR $\left(\mathrm{CD}_{3} \mathrm{OD}\right)(\delta \mathrm{ppm}$ ): $8.5(1 \mathrm{H}, \mathrm{s}, \mathrm{ArH}), 8.4$ $(1 \mathrm{H}, \mathrm{d}, J=8.3 \mathrm{~Hz}, \mathrm{ArH}), 8.2(1 \mathrm{H}, \mathrm{d}, J=9.0 \mathrm{~Hz}, \mathrm{ArH}), 7.9(1 \mathrm{H}, \mathrm{t}, J=7.6 \mathrm{~Hz}, \mathrm{ArH}), 7.7(1 \mathrm{H}, \mathrm{d}, J=8.3 \mathrm{~Hz}$, $\mathrm{ArH}), 7.6(1 \mathrm{H}, \mathrm{t}, J=8.1 \mathrm{~Hz}, \mathrm{ArH}), 7.0(1 \mathrm{H}, \mathrm{d}, J=9.0 \mathrm{~Hz}, \mathrm{ArH}), 4.0\left(2 \mathrm{H}, \mathrm{t}, J=5.9 \mathrm{~Hz}, \mathrm{CH}_{2}\right), 3.8(2 \mathrm{H}$, t, $\left.J=5.9 \mathrm{~Hz}, \mathrm{CH}_{2}\right), 3.5\left(2 \mathrm{H}, \mathrm{t}, J=7.1 \mathrm{~Hz}, \mathrm{CH}_{2}\right), 3.1\left(2 \mathrm{H}, \mathrm{t}, J=7.8 \mathrm{~Hz}, \mathrm{CH}_{2}\right), 2.3(2 \mathrm{H}, \mathrm{p}, J=7.6$, $\left.7.6 \mathrm{~Hz}, \mathrm{CH}_{2}\right)$, IR $(\mathrm{KBr}) \vee\left(\mathrm{cm}^{-1}\right): 1585.7,1646.1,2852.8,2928.0$, 3435.6; MS (FAB) $\mathrm{m} / z(\mathrm{M}+1)$ 363.2, 348.2; 185.0; MS-HR (FAB) Calc. for $\mathrm{C}_{20} \mathrm{H}_{22} \mathrm{~N}_{6} \mathrm{O}: 362.18551$ Found: $363.19414(\mathrm{M}+1)$.

3.1.21. 6-Hydrazino- $N$-[3-(2,3-dihydro-1H-cyclopenta[b]quinolin-9-ylamino)propyl]nicotinamide hydrochloride (6b)

A 5b $(0.20 \mathrm{~g}, 0.42 \mathrm{mmol})$ were combined as above to afford $\mathbf{6 b}$ as brown solid. Compound $\mathbf{6 b}$ : yield 36\%; mp 226-229 ${ }^{\circ} \mathrm{C}$; ${ }^{1} \mathrm{H}$ NMR (DMSO) ( $\delta$ ppm.): $14.1(1 \mathrm{H}, \mathrm{s}, \mathrm{HCl}), 9.9(1 \mathrm{H}, \mathrm{s}, \mathrm{NH}), 8.9$ $(1 \mathrm{H}, \mathrm{m}, \mathrm{ArH}), 8.8(1 \mathrm{H}, \mathrm{m}, \mathrm{ArH}), 8.5(1 \mathrm{H}, \mathrm{d}, J=8.7 \mathrm{~Hz}, \mathrm{ArH}), 8.1(1 \mathrm{H}, \mathrm{d}, J=8.9 \mathrm{~Hz}, \mathrm{ArH}), 7.8(1 \mathrm{H}, \mathrm{t}$, $J=7.3 \mathrm{~Hz}, \mathrm{ArH}), 7.6(1 \mathrm{H}, \mathrm{t}, J=7.7 \mathrm{~Hz}, \mathrm{ArH}), 6.9(1 \mathrm{H}, \mathrm{d}, J=8.5 \mathrm{~Hz}, \mathrm{ArH}), 3.7(4 \mathrm{H}, \mathrm{m}, \mathrm{NH}), 3.4-3.6$ $\left(4 \mathrm{H}, \mathrm{m}, \mathrm{CH}_{2}\right), 3.3\left(2 \mathrm{H}, \mathrm{t}, J=6.9 \mathrm{~Hz}, \mathrm{CH}_{2}\right), 3.1\left(2 \mathrm{H}, \mathrm{t}, J=8.3 \mathrm{~Hz}, \mathrm{CH}_{2}\right), 2.1\left(2 \mathrm{H}, \mathrm{m}, \mathrm{Hz}, \mathrm{CH}_{2}\right), 1.9$ $\left(2 \mathrm{H}, \mathrm{m}, \mathrm{CH}_{2}\right)$; IR $(\mathrm{KBr}) \vee\left(\mathrm{cm}^{-1}\right): 1585.3,1648.5,2857.0,2933.0,3277.6$; MS (FAB) $\mathrm{m} / z(\mathrm{M}+1)$ 377.2, 362.1; 185.0 MS-HR (FAB) Calc. for $\mathrm{C}_{21} \mathrm{H}_{24} \mathrm{~N}_{6} \mathrm{O}: 376.20116$ Found: $377.21072(\mathrm{M}+1)$. 
3.1.22. 6-Hydrazino- $N$-[4-(2,3-dihydro- $1 H$-cyclopenta[b]quinolin-9-ylamino)butyl]nicotinamide hydrochloride $(\mathbf{6 c})$

A $5 \mathbf{c}(0.20 \mathrm{~g}, 0.41 \mathrm{mmol})$ were combined as above to afford $\mathbf{6 c}$ as yellow solid. Compound $\mathbf{6 c}$ : yield 30\%; mp 171-173 ${ }^{\circ} \mathrm{C} ;{ }^{1} \mathrm{H}$ NMR (DMSO) $(\delta \mathrm{ppm}$ ): $14.3(1 \mathrm{H}, \mathrm{s}, \mathrm{HCl}), 9.9(1 \mathrm{H}, \mathrm{s}, \mathrm{NH}), 8.8(1 \mathrm{H}, \mathrm{m}$, $\operatorname{ArH}), 8.7(1 \mathrm{H}, \mathrm{m}, \mathrm{ArH}), 8.6(1 \mathrm{H}, \mathrm{m}, \mathrm{ArH}), 8.1(1 \mathrm{H}, \mathrm{d}, J=8.9 \mathrm{~Hz}, \mathrm{ArH}), 7.8(1 \mathrm{H}, \mathrm{t}, J=8.3 \mathrm{~Hz}, \mathrm{ArH})$, $7.6(1 \mathrm{H}, \mathrm{t}, J=6.7 \mathrm{~Hz}, \mathrm{ArH}), 6.9(1 \mathrm{H}, \mathrm{d}, J=8.5 \mathrm{~Hz}, \mathrm{ArH}), 3.7(4 \mathrm{H}, \mathrm{m}, \mathrm{NH}), 3.3-3.4\left(4 \mathrm{H}, \mathrm{m}, \mathrm{CH}_{2}\right), 3.2$ $\left(2 \mathrm{H}, \mathrm{t}, J=7.7 \mathrm{~Hz}, \mathrm{CH}_{2}\right), 3.1\left(2 \mathrm{H}, \mathrm{t}, J=7.7 \mathrm{~Hz}, \mathrm{CH}_{2}\right), 2.1\left(2 \mathrm{H}, \mathrm{m}, \mathrm{CH}_{2}\right), 1.6-1.8\left(4 \mathrm{H}, \mathrm{m}, \mathrm{CH}_{2}\right)$; IR $(\mathrm{KBr}) \vee\left(\mathrm{cm}^{-1}\right): 1541.7,1647.7,2857.4,2904.7,3414.7$; MS (FAB) $m / z(\mathrm{M}+1)$ 391.4, 376.4, 185.0; MS-HR (FAB) Calc. for $\mathrm{C}_{22} \mathrm{H}_{26} \mathrm{~N}_{6} \mathrm{O}: 391.21681$ Found: $391.22435(\mathrm{M}+1)$.

3.1.23. 6-Hydrazino- $N$-[5-(2,3-dihydro- $1 H$-cyclopenta[b]quinolin-9-ylamino)pentyl]nicotinamide hydrochloride (6d)

A 5d $(0.20 \mathrm{~g}, 0.40 \mathrm{mmol})$ were combined as above to afford $\mathbf{6 d}$ as brown solid. Compound $\mathbf{6 d}$ : yield 36\%; mp 107-108 ${ }^{\circ} \mathrm{C} ;{ }^{1} \mathrm{H}$ NMR (DMSO) $(\delta \mathrm{ppm}$.):14.4 (1H, s, HCl), $10.0(1 \mathrm{H}, \mathrm{s}, \mathrm{NH}), 8.9$ $(1 \mathrm{H}, \mathrm{m}, \mathrm{ArH}), 8.8(2 \mathrm{H}, \mathrm{m}, \mathrm{ArH}), 8.1(1 \mathrm{H}, \mathrm{d}, J=8.9 \mathrm{~Hz}, \mathrm{ArH}), 7.8(1 \mathrm{H}, \mathrm{t}, J=10.1 \mathrm{~Hz}, \mathrm{ArH}), 7.6(1 \mathrm{H}$, $\mathrm{t}, J=7.5 \mathrm{~Hz}, \mathrm{ArH}), 6.9(1 \mathrm{H}, \mathrm{d}, J=8.9 \mathrm{~Hz}, \mathrm{ArH}), 3.7(4 \mathrm{H}, \mathrm{m}, \mathrm{NH}), 3.3-3.4\left(4 \mathrm{H}, \mathrm{m}, \mathrm{CH}_{2}\right), 3.2(2 \mathrm{H}, \mathrm{t}$, $\left.J=9.9 \mathrm{~Hz}, \mathrm{CH}_{2}\right), 3.1\left(2 \mathrm{H}, \mathrm{t}, J=7.5 \mathrm{~Hz}, \mathrm{CH}_{2}\right), 2.1\left(2 \mathrm{H}, \mathrm{p}, J=7.5,7.1 \mathrm{~Hz}, \mathrm{CH}_{2}\right), 1.4-1.6\left(6 \mathrm{H}, \mathrm{m}, \mathrm{CH}_{2}\right)$; IR $(\mathrm{KBr}) \vee\left(\mathrm{cm}^{-1}\right): 1558.4,1647.7,2858.4,2931.2,3385.2$; MS (FAB) $m / z(\mathrm{M}+1) 405.3,390.3$, 185.0; MS-HR (FAB) Calc. for $\mathrm{C}_{23} \mathrm{H}_{28} \mathrm{~N}_{6} \mathrm{O}: 404.23246$ Found: $405.24165(\mathrm{M}+1)$.

3.1.24. 6-Hydrazino- $N$-[6-(2,3-dihydro- $1 H$-cyclopenta[ $b]$ quinolin-9-ylamino)hexyl]nicotinamide hydrochloride (6e)

A 5e $(0.20 \mathrm{~g}, 0.39 \mathrm{mmol})$ were combined as above to afford $\mathbf{6 e}$ as yellow solid. Compound $\mathbf{6 e}$ : yield 33\%; mp 166-167 ${ }^{\circ} \mathrm{C} ;{ }^{1} \mathrm{H}$ NMR (DMSO) $(\delta \mathrm{ppm}):. 14.4(1 \mathrm{H}, \mathrm{s}, \mathrm{HCl}), 10.0(1 \mathrm{H}, \mathrm{s}, \mathrm{NH}), 8.9(1 \mathrm{H}, \mathrm{m}$, $\operatorname{ArH}), 8.8(2 \mathrm{H}, \mathrm{m}, \operatorname{ArH}), 8.1(1 \mathrm{H}, \mathrm{d}, J=6.7 \mathrm{~Hz}, \operatorname{ArH}), 7.9(1 \mathrm{H}, \mathrm{t}, J=8.1 \mathrm{~Hz}, \operatorname{ArH}), 7.6(1 \mathrm{H}, \mathrm{t}$, $J=8.1 \mathrm{~Hz}, \mathrm{ArH}), 6.9(1 \mathrm{H}, \mathrm{d}, J=8.9 \mathrm{~Hz}, \mathrm{ArH}), 3.7(4 \mathrm{H}, \mathrm{m}, \mathrm{NH}), 3.4-3.5\left(4 \mathrm{H}, \mathrm{m}, \mathrm{CH}_{2}\right), 3.3(2 \mathrm{H}, \mathrm{t}$, $\left.J=7.1 \mathrm{~Hz}, \mathrm{CH}_{2}\right), 3.1\left(2 \mathrm{H}, \mathrm{t}, J=7.7 \mathrm{~Hz}, \mathrm{CH}_{2}\right), 2.2\left(2 \mathrm{H}, \mathrm{p}, J=7.7,7.1 \mathrm{~Hz}, \mathrm{CH}_{2}\right), 1.4-1.7\left(8 \mathrm{H}, \mathrm{m}, \mathrm{CH}_{2}\right)$; IR $(\mathrm{KBr}) \vee\left(\mathrm{cm}^{-1}\right): 1559.3,1636.9,2857.4,2930.2,3424.8$; MS (FAB) $m / z(\mathrm{M}+1) 419.3,404.3$, 185.0; MS-HR (FAB) Calc. for $\mathrm{C}_{24} \mathrm{H}_{30} \mathrm{~N}_{6} \mathrm{O}: 418.24811$ Found: $419.25754(\mathrm{M}+1)$.

3.1.25. 6-Hydrazino- $N$-[7-(2,3-dihydro- $1 H$-cyclopenta[b]quinolin-9-ylamino)heptyl]nicotinamide hydrochloride (6f)

A $5 \mathbf{f}(0.20 \mathrm{~g}, 0.38 \mathrm{mmol})$ were combined as above to afford $\mathbf{6 f}$ as brown solid. Compound $\mathbf{6 f}$ : yield 43\%; mp 105-107 ${ }^{\circ} \mathrm{C} ;{ }^{1} \mathrm{H}$ NMR (DMSO) $(\delta \mathrm{ppm}$.):14.4 (1H, s, HCl), $10.0(1 \mathrm{H}, \mathrm{s}, \mathrm{NH}), 8.8(1 \mathrm{H}, \mathrm{m}$, $\operatorname{ArH}), 8.6(2 \mathrm{H}, \mathrm{m}, \operatorname{ArH}), 8.1(1 \mathrm{H}, \mathrm{d}, J=6.5 \mathrm{~Hz}, \operatorname{ArH}), 7.9(1 \mathrm{H}, \mathrm{t}, J=9.5 \mathrm{~Hz}, \operatorname{ArH}), 7.6(1 \mathrm{H}, \mathrm{t}$, $J=6.7 \mathrm{~Hz}, \mathrm{ArH}), 6.9(1 \mathrm{H}, \mathrm{d}, J=8.9 \mathrm{~Hz}, \mathrm{ArH}), 3.7(4 \mathrm{H}, \mathrm{m}, \mathrm{NH}), 3.4\left(4 \mathrm{H}, \mathrm{m}, \mathrm{CH}_{2}\right), 3.2(2 \mathrm{H}, \mathrm{t}, J=7.5 \mathrm{~Hz}$, $\left.\mathrm{CH}_{2}\right), 3.1\left(2 \mathrm{H}, \mathrm{t}, J=7.7 \mathrm{~Hz}, \mathrm{CH}_{2}\right), 2.2\left(2 \mathrm{H}, \mathrm{p}, J=7.1,7.1 \mathrm{~Hz}, \mathrm{CH}_{2}\right), 1.3-1.7\left(10 \mathrm{H}, \mathrm{m}, \mathrm{CH}_{2}\right)$; IR $(\mathrm{KBr})$ $v\left(\mathrm{~cm}^{-1}\right):$ 1558.9, 1648.0, 2855.3, 2926.6, 3259.7, 3405.0; MS (FAB) $m / z(\mathrm{M}+1)$ 433.3, 418.3, 185.0; MS-HR (FAB) Calc. for $\mathrm{C}_{25} \mathrm{H}_{32} \mathrm{~N}_{6} \mathrm{O}: 432.26376$ Found: $433.27207(\mathrm{M}+1)$. 
3.1.26. 6-Hydrazino- $N$-[8-(2,3-dihydro- $1 H$-cyclopenta[b]quinolin-9-ylamino)octyl]nicotinamide hydrochloride $(\mathbf{6 g})$

A $5 \mathbf{g}(0.20 \mathrm{~g}, 0.37 \mathrm{mmol})$ were combined as above to afford $\mathbf{6 g}$ as yellow solid. Compound $\mathbf{6 g}$ : yield 43\%; mp 170-173 ${ }^{\circ} \mathrm{C} ;{ }^{1} \mathrm{H}$ NMR (DMSO) $(\delta \mathrm{ppm}$.): $14.2(1 \mathrm{H}, \mathrm{s}, \mathrm{HCl}), 9.9(1 \mathrm{H}, \mathrm{s}, \mathrm{NH}), 8.8(1 \mathrm{H}$, m, ArH), $8.6(2 \mathrm{H}, \mathrm{m}, \mathrm{ArH}), 8.1(1 \mathrm{H}, \mathrm{d}, J=8.9 \mathrm{~Hz}, \mathrm{ArH}), 7.8(1 \mathrm{H}, \mathrm{m}, \mathrm{ArH}), 7.6(1 \mathrm{H}, \mathrm{m}, \operatorname{ArH}), 6.9$ $(1 \mathrm{H}, \mathrm{d}, J=8.3 \mathrm{~Hz}, \mathrm{ArH}), 3.7(4 \mathrm{H}, \mathrm{m}, \mathrm{NH}), 3.3\left(4 \mathrm{H}, \mathrm{m}, \mathrm{CH}_{2}\right), 3.2\left(2 \mathrm{H}, \mathrm{t}, J=5.7 \mathrm{~Hz}, \mathrm{CH}_{2}\right), 3.1(2 \mathrm{H}, \mathrm{t}$, $\left.J=9.1 \mathrm{~Hz}, \mathrm{CH}_{2}\right), 2.2\left(2 \mathrm{H}, \mathrm{p}, J=7.3,7.3 \mathrm{~Hz}, \mathrm{CH}_{2}\right), 1.3-1.8\left(12 \mathrm{H}, \mathrm{m}, \mathrm{CH}_{2}\right)$; IR $(\mathrm{KBr}) v\left(\mathrm{~cm}^{-1}\right): 1558.5$, 1634.0, 2854.2, 2926.7, 3200.6, 3422.2; MS (FAB) $m / z(\mathrm{M}+1)$ 447.1, 432.2, 185.0; MS-HR (FAB) Calc. for $\mathrm{C}_{26} \mathrm{H}_{34} \mathrm{~N}_{6} \mathrm{O}: 446.27941$ Found: $447.28878(\mathrm{M}+1)$.

3.1.27. 6-Hydrazino- $N$-[9-(2,3-dihydro-1H-cyclopenta[ $b]$ quinolin-9-ylamino)nonyl]nicotinamide hydrochloride (6h)

A $5 \mathbf{h}(0.20 \mathrm{~g}, 0.35 \mathrm{mmol})$ were combined as above to afford $\mathbf{6 h}$ as brown solid. Compound $\mathbf{6 h}$ : yield 44\%; mp 85-87 ${ }^{\circ} \mathrm{C} ;{ }^{1} \mathrm{H}$ NMR (DMSO) $(\delta \mathrm{ppm}$.): $14.5(1 \mathrm{H}, \mathrm{s}, \mathrm{HCl}), 10.0(1 \mathrm{H}, \mathrm{s}, \mathrm{NH}), 8.9(1 \mathrm{H}, \mathrm{m}$, $\operatorname{ArH}), 8.8(2 \mathrm{H}, \mathrm{m}, \operatorname{ArH}), 8.1(1 \mathrm{H}, \mathrm{d}, J=6.9 \mathrm{~Hz}, \operatorname{ArH}), 7.8(1 \mathrm{H}, \mathrm{t}, J=6.7 \mathrm{~Hz}, \operatorname{ArH}), 7.6(1 \mathrm{H}, \mathrm{t}$, $J=7.1 \mathrm{~Hz}, \mathrm{ArH}), 6.9(1 \mathrm{H}, \mathrm{d}, J=8.9 \mathrm{~Hz}, \mathrm{ArH}), 3.7(4 \mathrm{H}, \mathrm{m}, \mathrm{NH}), 3.4\left(4 \mathrm{H}, \mathrm{m}, \mathrm{CH}_{2}\right), 3.3\left(2 \mathrm{H}, \mathrm{m}, \mathrm{CH}_{2}\right)$, $3.2\left(2 \mathrm{H}, \mathrm{t}, J=7.7 \mathrm{~Hz}, \mathrm{CH}_{2}\right), 2.1\left(2 \mathrm{H}, \mathrm{m}, \mathrm{CH}_{2}\right), 1.5-1.7\left(14 \mathrm{H}, \mathrm{m}, \mathrm{CH}_{2}\right)$; IR $(\mathrm{KBr}) v\left(\mathrm{~cm}^{-1}\right): 1585.8$, 1647.3, 2853.5, 2925.4, 3438.1; MS (FAB) $m / z(\mathrm{M}+1)$ 461.3, 446.3, 183.0; MS-HR (FAB) Calc. for $\mathrm{C}_{27} \mathrm{H}_{36} \mathrm{~N}_{6} \mathrm{O}: 460.29506$ Found: $461.30121(\mathrm{M}+1)$.

\subsection{Biochemical Studies}

Determination of the inhibitory activity of all synthesized compounds towards AChE and BChE was performed by means of Ellman's spectrophotometric method with our own modifications. Every sample contained 5,5'-dithiobisnitrobenzoic acid (DTNB, $0.05 \mathrm{~mL}, 0.5 \mathrm{M}$ ), acetylthiocholine iodide (substrate), newly synthesized inhibitor, and AChE (5 units $/ \mathrm{mL}$ ) or BChE (5 units $/ \mathrm{mL}$ ), respectively, in cholinesterases activity measurements. Every measurement was conducted in the presence of phosphate buffer $(0.1 \mathrm{M}, \mathrm{pH} 8.0)$ at $37{ }^{\circ} \mathrm{C}$. The total volume of every sample amounted to $3 \mathrm{~mL}$. Measurement procedure was identical for all samples. All ingredients were incubated for $1 \mathrm{~min}$ and then the absorbance was recorded at $412 \mathrm{~nm}$. Seven concentrations of acetylthiocholine iodide were used in order to obtain the inhibition curves for every compound. Every measurement was conducted three times. Also, samples without inhibitor were evaluated in order to obtain absolute AChE and $\mathrm{BChE}$ activity. The value of $\mathrm{IC}_{50}$, defined as the drug concentration that contributes to the inhibition of $50 \% \mathrm{AChE}$ or BChE activity, was determined by non-linear and linear regression.

All reagents: DTNB, enzymes (C2629 Acetylcholinesterase from Electrophorus electricus (electric eel) and C4290 Butyrylcholinesterase from equine serum) and acetylthiocholine iodide were purchased from Sigma-Aldrich.

\subsection{Molecular Modeling}

The three-dimensional structures of inhibitors were created by Corina on-line (Molecular Networks) and subsequently prepared with Sybyl 8.0 (Tripos). Atom types were checked, hydrogen atoms were 
added, and, then, Gasteiger-Marsili charges were assigned. Ligands were bound to acetylcholinesterase from 2CKM and butyrylcholinesterase from 1P0I crystal complex. Protein was prepared before binding with GoldSuite 5.0.1 (CCDC). All histidine residues were protonated at $\mathrm{N} \varepsilon$, the hydrogen atoms were added, ligand and water molecules were removed, and the binding site was defined as all amino acid residues within $10 \AA$ from bis-(7)-tacrine for AChE and $20 \AA$ from the glycerol molecule present in the active center of BChE. A standard set of genetic algorithms with population size of 100 , number of operations 100,000 , and clustering with a tolerance of $1 \AA$ was applied. As a result 10 ligand poses, sorted by GoldScore (AChE) and ChemScore (BChE) function value were obtained. The results were visualized by PyMOL 0.99rc6 (DeLano Scientific LLC).

\subsection{Spectrophotometric Experiments}

The absorption spectrum was obtained by scanning the sample between 200 and $350 \mathrm{~nm}$ with a Perkin Elmer spectrophotometer. The experiment was started with a solution of pure water at room temperature. The stability of compound $\mathbf{6 a}$ was assessed by monitoring the variability of the spectrum at regular intervals (15 min) over $4 \mathrm{~h}$.

\subsection{Radiolabelling}

For radiolabeling with Tc-99m, $1 \mathrm{mg}$ of the ligand 6a was dissolved in $150 \mu \mathrm{L}$ of water. Subsequently, $100 \mathrm{mg}$ of tricine and $1.5 \mathrm{~mL}$ of technetium eluate were added together with $25 \mu \mathrm{L}$ of $\mathrm{SnCl}_{2}$ in ethanol $(1 \mathrm{mg} / \mathrm{mL})$. After $30 \mathrm{~min}$ incubation at room temperature, quality control was performed by HPLC analysis. HPLC analysis was performed on an Agilent System 1100 Series with UV and radiometric detection, with LiChrocart column 250-3 Luichrospher 100 RP-18 (5 $\mu \mathrm{m})$. Flow rate was $1 \mathrm{~mL} / \mathrm{min}$.

Gradient I:

Mobile phase A: $0.9 \% \mathrm{NaCl}, \mathrm{B}: \mathrm{CH}_{3} \mathrm{CN}$.

$1-25 \min 50 \% \mathrm{~B}$.

$25-30 \min 50 \%-100 \% \mathrm{~B}$.

30-35 min 100\% B.

$35-40 \min 100 \%-0 \% \mathrm{~B}$.

Gradient II:

$0-10 \min 0 \% \mathrm{~B}$.

$10-25 \min 0 \%-100 \% \mathrm{~B}$.

25-30 min 100\% B.

$30-35 \min 100 \%-0 \% \mathrm{~B}$.

\subsection{Biodistribution Studies in Rats}

\subsubsection{Animals}

For biological experiments, the radiolabelled compound was dissolved in saline to a concentration of the ligand equal to $100 \mu \mathrm{g} / \mathrm{mL}$. Intravenous dose was $20 \mu \mathrm{g}$ per animal 
For biodistribution studies, male Wistar rats weighing 190-260 g were used. Prior to the experiment, the animals were fasted overnight (to empty the bowels), but had free access to water. All animal experiments were approved by the Ethics Committee of the Faculty of Pharmacy, Charles University, Hradec Kralove.

\subsubsection{Biodistribution in Rats}

The agent was administered to rats intravenously in a volume of $0.2 \mathrm{~mL}$. During the course of the experiments, each animal was placed in an individual cage. At various time points after injection, the carotid artery was exposed under ether anesthesia and a blood sample was collected in glass tubes containing dry heparin. The rats were sacrificed and dissected. The organs of interest were weighed and counted for radioactivity in an automatic gamma counter (1480 Wizard 3).

The results were expressed as mean \pm standard deviations of at least four animals.

\section{Conclusions}

As a consequence of a limited number of efficacious drugs in the treatment of AD, many scientific teams are aiming to discover novel compounds able to improve cholinergic neurotransmission. Currently approved AChE inhibitors, such as galantamine, rivastigmine, and donepezil, provide comparatively little chance for a prolonged improvement in cognitive functions. Thus, the search for novel compounds with anticholinesterase activity continues as numerous scientists are focused on the development of novel compounds such as cystamine-tacrine dimers [37], bisquaternary isoquinolinium derivatives [38], diversely substituted furo[2,3-b]quinolin-4-amine and pyrrolo[2,3-b]quinolin-4-amine derivatives [39], and tacrine-8-hydroxyquinoline hybrid [40] that might increase the level of ACh.

Several teams have also utilized derivatives of tacrine as potential radiopharmaceuticals. Tacrine and its modified structure derivatives were marked with radioactive isotope in order to determine the level of $\mathrm{AChE}$ or $\mathrm{BChE}$ as a function of tacrine analogue labeled isotope accumulated in the target site $[41,42]$. Also, other AChE inhibitors such as donepezil and huperzine were modified and marked with radioisotopes $[43,44]$.

In our previous papers, we presented the synthesis and biological evaluation of derivatives of tetrahydroacridine derivatives with hydrazine nicotinate (HYNIC) moiety $[45,46]$. The fragment of tetrahydroacridine that possesses the possibility to inhibit both the moieties of cholinesterase and HYNIC has the potential to be utilized as a co-ligand for radiolabeling.

As described in this article, eight synthesized compounds differed from each other only in the length of the aliphatic chain between the tetrahydroacridine and the hydrazine nicotinate moiety. According to the results of the studies, compounds showing the highest activity with regard to AChE inhibition were those with six and eight carbon atoms in the aliphatic chain; which were approximately 7- and 9- fold more active than tacrine, respectively. This data suggests that altering the molecule by incorporating a longer aliphatic chain proved to be a good choice. All novel compounds, apart from the one with six carbon atoms in the aliphatic chain, described in these studies were characterized, in comparison to tacrine, by lower inhibitory activity towards BChE [45,46].

Within this work, compared to our previous studies, the six membered ring of tetrahydroacridine was exchanged with a five membered ring; this influenced the activity and interaction with active sites. 
Of all the synthesized compounds, the most active was $6 \mathbf{h}\left(\mathrm{IC}_{50}=3.65 \mathrm{nM}\right)$. This compound was about 1.5-fold more active than tacrine and, as compared to this reference compound, more selective towards AChE. Compound $\mathbf{6 g}$ exhibited a similar value of $\mathrm{IC}_{50}$ to that of tacrine. Similarly to our previous studies, obtained data suggests that the activity of the synthesized compounds increases simultaneously with the length of the aliphatic chain between the hydrazine nicotinate moiety and 2,3-dihydro- $1 H$-cyclopenta[$[b]$ quinolone. Our data showed that all synthesized molecules were characterized by lower $\mathrm{BChE}$ inhibitory activity in comparison to tacrine.

On the other hand, selectivity of the obtained compounds is very promising because the moiety of 6-hydrazinenicotinic acid (HYNIC) is thought to be responsible for the binding of technetium-99m radiotracer $\left({ }^{99 \mathrm{~m}} \mathrm{Tc}\right.$ ) (as reported previously by Abrams et al.) [26].

According to molecular modeling studies, all ligands were extended along the active gorge and interacted with both the catalytic and peripheral site of AChE. This dual type of binding to AChE is responsible for an additional function related to the interaction with $\beta$-amyloid. The mode of binding with BChE was similar; the main difference was the location of the hydrazinenicotinic fragment in the reduced peripheral anionic site of $\mathrm{BChE}$. These results are of vital importance as it has been established that AChE not only plays a crucial role in cholinergic dysfunction, but also is involved in the $\beta$-amyloid cascade via the AChE peripheral anionic site (PAS), such as mediating the adhesion, differentiation and deposition of $\beta$-amyloid in $\mathrm{AD}$. It has been reported that $\mathrm{AChE}$ is a protein associated with the amyloid core of mature senile plaques, pre-amyloid diffuse deposits, and cerebral blood vessels in AD brain. Alvares et al., in in vitro studies showed that $\mathrm{AChE}$ is incorporated into $\beta$-amyloid aggregates by forming macromolecular complexes with the growing $\beta$-amyloid fibrils. The following scientific work of Alvares demonstrated that these complexes accelerate the maturation of $\beta$-amyloid plaques and are more toxic at the cellular level than the amyloid fibrils alone $[47,48]$. Molecular modeling studies presented within this work revealed that synthesized compounds are dual binding site inhibitors; thus, there is a possibility to influence non-cholinergic functions of $\mathrm{AChE}$ including AChE-induced aggregation of $\beta$-amyloid.

Biodistribution studies in rats revealed that compound 6a exhibited comparatively rapid blood radioactivity clearance. ${ }^{99 \mathrm{~m}}$ Tc-radioactivity was mainly located in the liver, and to a lesser extent in the kidney, lung, and the gastrointestinal tract. Low radioactivity concentrations in the brain suggest that these agents do not cross the blood-brain barrier, and, therefore, cannot be regarded as potential agents for diagnosis of Alzheimer's disease. However, similarly to $\left[{ }^{11} \mathrm{C}\right]$ choline, the synthesized compounds might be further evaluated as molecules suitable for the detection of cancers in certain organs (e.g., liver, kidney, lungs) or to monitor the response to various therapies. For example, there are scientific reports which prove that $\left[{ }^{11} \mathrm{C}\right]$ choline, developed by Hara et al., as an oncologic PET (positron emission tomography) radiopharmaceutical, might be utilized with good results in the diagnosis of lung cancer [49], colon cancer [50], or prostate cancer [51]. Furthermore, $\left[{ }^{11} \mathrm{C}\right]$ choline and multimodality fusion imaging with integrated PET and contrast-enhanced CT (PET/CT) could be used to monitor the response to anti-androgenic therapy [52].

Results of our synthesis and analysis suggest that the obtained hybrids of 2,3-dihydro- $1 H$-cyclopenta[ $b]$ quinolone and 6-hydrazinonicotynic acid may be considered as novel potential anti-Alzheimer's drugs. Conversely, these compounds, following radiolabeling, could be 
used in the detection of the cholinergic deficit occurring in the peripheral nervous system or in the diagnosis of various types of cancers or to correct physiological functions.

\section{Acknowledgments}

This work was supported by the grant (N N405 669940) from National Science Centre in Poland and by the Grant Agency of the Czech Republic (grant P304/10/1738).

\section{References}

1. Delfini, M.; di Coco, M.E.; Piccioni, F.; Porcelli, F.; Borioni, A.; Rodomonte, A.; del Giudice, M.R. Tacrine derivatives-acetylcholinesterase interaction. 1H NMR relaxation study. Bioorg. Chem. 2007, 35, 243-257.

2. Kurz, A.; Perneczky, R. Novel insights for the treatment of Alzheimer's disease. Prog. Neuro-Psychopharmacol. 2011, 35, 373-379.

3. Sjogren, M.; Andreasen, N.; Blennow, K. Advances in the detection of Alzheimer's disease-use of cerebrospinal fluid biomarkers. Clin. Chim. Acta 2003, 332, 1-10.

4. Silmana, I.; Sussman, J.L. Acetylcholinesterase: How is structure related to function? Chem. Biol. Interact. 2008, 175, 3-10.

5. Soreq, H.; Seidman, S. Acetylcholinesterase-New roles for an old actor. Nat. Rev. Neurosci. 2001, 2, 294-302.

6. Akasofu, S.; Kimura, M.; Kosasa, T.; Sawada, K.; Ogura, H. Study of neuroprotection of donepezil, a therapy for Alzheimer's disease. Chem. Biol. Interact. 2008, 175, 222-226.

7. Jia, P.; Sheng, R.; Zhang, J.; Fang, L.; He, Q.; Yang, B.; Hu, Y. Design, synthesis and evaluation of galanthamine derivatives as acetylcholinesterase inhibitors. Eur. J. Med. Chem. 2009, 44, 772-784.

8. Pan, L.; Tan, J.-H.; Hou, J.-Q.; Huang, S.-L.; Gu, L.-Q.; Huang, Z.-S. Design, synthesis and evaluation of isaindigotone derivatives as acetylcholinesterase and butyrylcholinesterase inhibitors. Bioorg. Med. Chem. Lett. 2008, 18, 3790-3794.

9. Blass, J.P. Alzheimer's disease and Alzheimer's dementia: Distinct but overlapping entities. Neurobiol. Aging 2002, 23, 1077-1084.

10. Shah, R.S.; Lee, H.G.; Xiongwei, Z.; Perry, G.; Smith, M.A.; Castellani, R.J. Current approaches in the treatment of Alzheimer's disease. Biomed. Pharmacother. 2008, 62, 199-207.

11. Salloway, S.; Mintzer, J.; Weiner, M.F.; Cummings, J.L. Disease-modifying therapies in Alzheimer's disease. Alzheimer's Dement. 2008, 4, 65-79.

12. Jin, K.; Xie, L.; Mao, X.O.; Greenberg, D.A. Alzheimer's disease drugs promote neurogenesis. Brain Res. 2006, 1085, 183-188.

13. Liston, D.R.; Nielsen, J.A.; Villalobos, A.; Chapin, D.; Jones, S.B.; Hubbard, S.T.; Shalaby, I.A.; Ramirez, A.; Nason, D.; White, W.F. Pharmacology of selective acetylcholinesterase inhibitors: Implications for use in Alzheimer's disease. Eur. J. Pharmacol. 2004, 486, 9-17.

14. Davis, K.L.; Pochwik, P. Tacrine. Lancet 1995, 11, 625-630.

15. Musiał, A.; Bajda, M.; Malawska, B. Development of acetylcholinesterase inhibitors for Alzheimer's disease treatment. Curr. Med. Chem. 2007, 14, 2654-2679. 
16. Tumiatti, V.; Minarini, A.; Bolognesi, M.L.; Milelli, A.; Rosini, M.; Melchiorre, C. Tacrine derivatives and Alzheimer's disease. Curr. Med. Chem. 2010, 17, 1825-1838.

17. Hu, M.-K.; Wu, L.-J.; Hsiao, G.; Yen, M.-H. Homodimeric tacrine congeners as acetylcholinesterase inhibitors. J. Med. Chem. 2002, 45, 2277-2282.

18. Rydberg, E.H.; Brumshtein, B.; Greenblatt, H.M.; Wong, D.M.; Shaya, D.; Williams, L.D.; Carlier, P.R.; Pang, Y.-P.; Silman, I.; Sussman, J.L. Complexes of alkylene-linked tacrine dimers with Torpedo californica acetylcholinesterase: Binding of bis(5)-tacrine produces a dramatic rearrangement in the active-site gorge. J. Med. Chem. 2006, 49, 5491-5500.

19. Li, W.; Mak, M.; Jiang, H.; Wang, Q.; Pang, Y.; Chen, K.; Han, Y. Novel anti-Alzheimer's dimer Bis(7)-cognitin: Cellular and molecular mechanisms of neuroprotection through multiple targets. Neurotherapeutics 2009, 6, 187-201.

20. Carlier, P.R.; Han, Y.F.; Chow, E.S.; Li, C.P.; Wang, H.; Lieu, T.X.; Wong, H.S.; Pang, Y.P. Evaluation of short-tether Bis-THA AChE inhibitors. A further test of the dual binding site hypothesis. Bioorg. Med. Chem. 1999, 7, 351-357.

21. Shen, Y.; Yu, Y.; Lv, H.; Feng, L.; Zhang, G. Design, synthesis and evaluation of tacrine based acetylcholinesterase inhibitors. Lett. Drug Des. Discov. 2010, 7, 341-345.

22. Zhou, J.; Hu, X.; Zhang, H.; Qian, H.; Huang, W.; Qi, F.; Zhang, Y. Synthesis and biological evaluation of 5,6-dihydro-benzo[c]acridin-7-ol derivatives as anti-Alzheimer's disease drugs. Lett. Drug Des. Discov. 2009, 6, 623-628.

23. Belluti, F.; Piazzi, L.; Bisi, A.; Gobbi, S.; Bartolini, M.; Cavalli, A.; Valenti, P.; Rampa, A. Design, synthesis, and evaluation of benzophenone derivatives as novel acetylcholinesterase inhibitors. Eur. J. Med. Chem. 2009, 44, 1341-1348.

24. Tomassoli, I.; Ismaili, L.; Pudlo, M.; de los Ríos, C.; Soriano, E.; Colmena, I.; Gandía, L.; Rivas, L.; Samadi, A.; Marco-Contelles, J.; et al. Synthesis, biological assessment and molecular modeling of new dihydroquinoline-3-carboxamides and dihydroquinoline-3-carbohydrazide derivatives as cholinesterase inhibitors, and Ca channel antagonists. Eur. J. Med. Chem. 2011, 46, 1-10.

25. Samadi, A.; Valderas, C.; de los Ríos, C.; Bastida, A.; Chioua, M.; González-Lafuente, L.; Colmena, I.; Gandía, L.; Romero, A.; del Barrio, L.; et al. Cholinergic and neuroprotective drugs for the treatment of Alzheimer and neuronal vascular diseases. II. Synthesis, biological assessment, and molecular modelling of new tacrine analogues from highly substituted 2-aminopyridine-3-carbonitriles. Bioorg. Med. Chem. 2011, 19, 122-133.

26. Abrams, M.J.; Juweid, M.; TenKate, C.I.; Schwartz, D.A.; Hauser, M.M.; Gaul, F.E. Technetium-99-m-Human Polyclonal IgG radiolabeled via the hydrazino nicotinamide derivative for imaging focal sites of infection in rats. J. Nucl. Med. 1990, 31, 2022-2028.

27. Dorronsoro, I.; Alonso, D.; Castro, A. Synthesis and biological evaluation of tacrine-thiadiazolidinone hybrids as dual acetylcholinesterase inhibitors. Arch. Pharm. Chem. Life Sci. 2005, 338, 18-23.

28. Rosini, M.; Andrisano, V.; Bartolini, M.; Bolognesi, M.L.; Hrelia, P.; Minarini, A.; Tarozzi, A.; Melchiorre, C. Rational approach to discover multipotent anti-Alzheimer drugs. J. Med. Chem. 2005, 48, 360-363.

29. Fang, L.; Kraus, B.; Lehman, J. Tacrine-Ferulic acid hybrids as multi-potent anti-Alzheimer drug candidates. Bioorg. Med. Chem. 2008, 18, 2905-2909. 
30. Carlier, P.R.; Chow, E.S.-W.; Han, Y.F.; Liu, J.; El Yazak, J.; Pang, Y.-P. Heterodimeric tacrine-based acetylcholinesterase inhibitors: Investigating ligand-peripheral site interactions. J. Med. Chem. 1999, 42, 4225-4231.

31. Fang, L.; Appenroth, D.; Decker, M.; Kiehntopf, M.; Lupp, A.; Peng, S.; Fleck, C.; Zhang, Y.; Lehmann, J. Tacrine hybrid compounds improve scopolamine-induced cognition impairment and show less hepatotoxicity. J. Med. Chem. 2008, 51, 7666-7669.

32. Ellman, G.L.; Courtney, K.D.; Andres, V.; Featherstone, R.M. A new and rapid colorimetric determination of acetylcholinesterase activity. Biochem. Pharm. 1961, 7, 88-95.

33. Cheng, Y.C.; Prusoff, W.H. Relationship between the inhibition constant (K1) and the concentration of inhibitor which causes 50 per cent inhibition (I50) of an enzymatic reaction. Biochem. Pharm. 1973, 22, 3099-3108.

34. Tipton, K.F. Commentary: Enzyme kinetics in relation to enzyme inhibitors. Biochem. Pharm. 1973, 22, 2933-2941.

35. Neşe Çokuğraş, A. Butyrylcholinesterase: Structure and physiological importance. Turk. J. Biochem. 2003, 28, 54-61.

36. Liu, S.; Edwards, D.S. 99mTc-Labeled small peptides as diagnostic radiopharmaceuticals. Chem. Rev. 1999, 99, 2235-2268.

37. Minarini, A.; Milelli, A.; Tumiatti, V.; Rosini, M.; Simoni, E.; Bolognesi, M.L.; Andrisano, V.; Bartolini, M.; Motori, E.; Angeloni, C.; et al. Cystamine-Tacrine dimer: A new multi-target-directed ligand as potential therapeutic agent for Alzheimer's disease treatment. Neuropharmacology 2012, 62, 997-1003.

38. Binder, J.; Paar, M.; Jun, D.; Pohanka, M.; Hrabinova, M.; Opletalova, V.; Kuca, K. New bisquaternary isoquinolinium inhibitors of brain cholinesterases-synthesis and anticholinesterase. Lett. Drug Des. Discov. 2010, 7, 1-4.

39. Martins, C.; Carmo Carreiras, M.; León, R.; de los Ríos, C.; Bartolini, M.; Andrisano, V.; Iriepa, I.; Moraleda, I.; Gálvez, E.; García, M.; et al. Synthesis and biological assessment of diversely substituted furo[2,3-b]quinolin-4-amine and pyrrolo[2,3-b]quinolin-4-amine derivatives, as novel tacrine analogues. Eur. J. Med. Chem. 2011, 46, 6119-6130.

40. Antequera, D.; Bolos, M.; Spuch, C.; Pascual, C.; Ferrer, I.; Fernandez-Bachiller, M.I.; Rodríguez-Franco, M.I.; Carro, E. Effects of a tacrine-8-hydroxyquinoline hybrid (IQM-622) on $\mathrm{A} \beta$ accumulation and cell death: Involvement in hippocampal neuronal loss in Alzheimer's disease. Neurobiol. Dis. 2012, 46, 682-691.

41. Akula, M.R.; Kabalka, G.W. Synthesis of 7-[123I]iodotacrine: A potential SPECT agent to map acetylcholinesterase. J. Labelled Compd. Radiopharm. 1999, 42, 959-964.

42. Nishioka, K.; Kamada, T.; Kanamaru, H. 14C-Labeling of a tetrahydroacridine, a novel CNS-selective cholinesterase inhibitor. J. Labelled Compd. Radiopharm. 1992, 31, 553-560.

43. Leman, L.; Kitson, S.L.; Brown, R.T.; Cairns, J.; Watters, W.; McMordie, A.; Murrell, V.; Marfurt, J. Synthesis of isotopically labelled [14C]ZT-1 (Debio-9902), [d3]ZT-1 and $(-)-[d 3]$ huperzine A, a new generation of acetylcholinesterase inhibitors. J. Labelled Compd. Radiopharm. 2011, 54, 720-730. 
44. De Vos, F.; Santens, P.; Vermeirsch, H.; Dewolf, I.; Dumont, F.; Slegers, G.; Dierckx, R.A.; de Reuck, J. Pharmacological evaluation of $\left[{ }^{11} \mathrm{C}\right]$ donepezil as tracer for visualization of acetylcholinesterase by PET. Nucl. Med. Biol. 2000, 27, 745-747.

45. Szymański, P.; Żurek, E.; Mikiciuk-Olasik, E. New tacrine-hydrazinonicotinamide hybrids as acetylcholinesterase inhibitors of potential interest for the early diagnostics of Alzheimer's disease. Pharmazie 2006, 61, 269-273.

46. Szymański, P.; Markowicz, M.; Mikiciuk-Olasik, E. Synthesis and biological activity of derivatives of tetrahydroacridine as acetylcholinesterase inhibitors. Bioorg. Chem. 2011, 39, 138-142.

47. Alvarez, A.; Opazo, C.; Alarcon, R.; Garrido, J.; Inestrosa, N.C. Acetylcholinesterase promotes the aggregation of amyloid- $\beta$-peptide fragments by forming a complex with the growing fibrils. J. Mol. Biol. 1997, 272, 348-361.

48. Alvarez, A.; Alarcon, R.; Opazo, C.; Campos, E.O.; Munoz, F.J.; Calderon, F.H.; Dajas, F.; Gentry, M.K.; Doctor, B.P.; de Mello, F.G.; et al. Stable complexes involving acetylcholinesterase and amyloid- $\beta$ peptide change the biochemical properties of the enzyme and increase the neurotoxicity of Alzheimer's fibrils. J. Neurosci. 1998, 18, 3213-3223.

49. Inagaki, K.; Morita, T.; Fujii, K.; Kosaka, N.; Hara, T. Mediastinal lymph node staging in non-small cell lung cancer using PET with C-11 choline and F-18 FDG. J. Nucl. Med. 1999, 40, $59-60$.

50. Hara, T.; Kosaka, N.; Kondo, T.; Kishi, H.; Kobori, O. Imaging of brain tumor, lung cancer, esophagus cancer, colon cancer, prostate cancer, and bladder cancer with [C-11]choline. J. Nucl. Med. 1997, 38, 250.

51. Reske, S.N.; Blumstein, N.M.; Neumaier, B.; Gottfried, H.W.; Finsterbusch, F.; Kocot, D.; Möller, P.; Glatting, G.; Perner, S. Imaging prostate cancer with 11C-choline PET/CT. J. Nucl. Med. 2006, 47, 1249-1254.

52. Giovacchini, G.; Picchio, M.; Coradeschi, E.; Scattoni, V.; Bettinardi, V.; Cozzarini, C.; Freschi, M.; Fazio, F.; Messa, C. [11C]Choline uptake with PET/CT for the initial diagnosis of prostate cancer: Relation to PSA levels, tumour stage and anti-androgenic therapy. Eur. J. Nucl. Med. Mol. Imaging 2008, 35, 1065-1073.

(C) 2012 by the authors; licensee MDPI, Basel, Switzerland. This article is an open access article distributed under the terms and conditions of the Creative Commons Attribution license (http://creativecommons.org/licenses/by/3.0/). 